\title{
Epithelial-Mesenchymal Transition (EMT) as a Therapeutic Target
}

\author{
Sven Jonckheere ${ }^{a, b}$ Jamie Adams ${ }^{c}$ Dominic De Groote ${ }^{a} \quad$ Kyra Campbell $^{c}$ \\ Geert Berx ${ }^{a, b}$ Steven Goossens ${ }^{a, d}$ \\ ${ }^{a}$ Cancer Research Institute Ghent (CRIG), Ghent University, Ghent, Belgium; bepartment of Biomedical Molecular \\ Biology, Ghent University, Ghent, Belgium; 'Department of Biomedical Science, The University of Sheffield, \\ Sheffield, UK; d Department of Diagnostic Sciences, Ghent University, Ghent, Belgium
}

\section{Keywords}

EMT · Therapy · Metastasis · Drug screening · Drosophila melanogaster $\cdot$ Zebrafish
EMT and discuss some already existing candidate drug targets and high-throughput screening tools to identify novel anti-EMT compounds.

(c) 2021 S. Karger AG, Base

\begin{abstract}
Metastasis is the spread of cancer cells from the primary tumour to distant sites and organs throughout the body. It is the primary cause of cancer morbidity and mortality, and is estimated to account for $90 \%$ of cancer-related deaths. During the initial steps of the metastatic cascade, epithelial cancer cells undergo an epithelial-mesenchymal transition (EMT), and as a result become migratory and invasive mesenchymal-like cells while acquiring cancer stem cell properties and therapy resistance. As EMT is involved in such a broad range of processes associated with malignant transformation, it has become an increasingly interesting target for the development of novel therapeutic strategies. AntiEMT therapeutic strategies could potentially not only prevent the invasion and dissemination of cancer cells, and as such prevent the formation of metastatic lesions, but also attenuate cancer stemness and increase the effectiveness of more classical chemotherapeutics. In this review, we give an overview about the pros and cons of therapies targeting
\end{abstract}

\section{Introduction}

An epithelial-mesenchymal transition (EMT) is a reversible physiological process, in which well-polarized epithelial cells lose their apical-basal polarity and their stable cell-cell contacts, while acquiring a more spindleshaped mesenchymal-like morphology with enhanced motile, migratory properties (Fig. 1) [Kalluri and Weinberg, 2009]. These morphological changes are associated with a repression of so-called epithelial markers (e.g., Ecadherin, claudins, occludins) and a gain of mesenchymal markers (e.g., vimentin, fibronectin, N-cadherin) [Lamouille et al., 2014; Nieto et al., 2016; Yang et al., 2020]. EMT was originally identified as a process important for the formation of the body plan and several tissues and organs during embryonic development [Thiery et al.,

Sven Jonckheere and Jamie Adams are co-first authors. karger@karger.com www.karger.com/cto

(C) 2021 S. Karger AG, Base

Karger!
Steven Goossens

Department of Diagnostic Sciences, Ghent University, MRB2 (entrance 38) Corneel Heymanslaan 10 BE-9000 Ghent (Belgium)

Steven.Goossens@ugent.be 


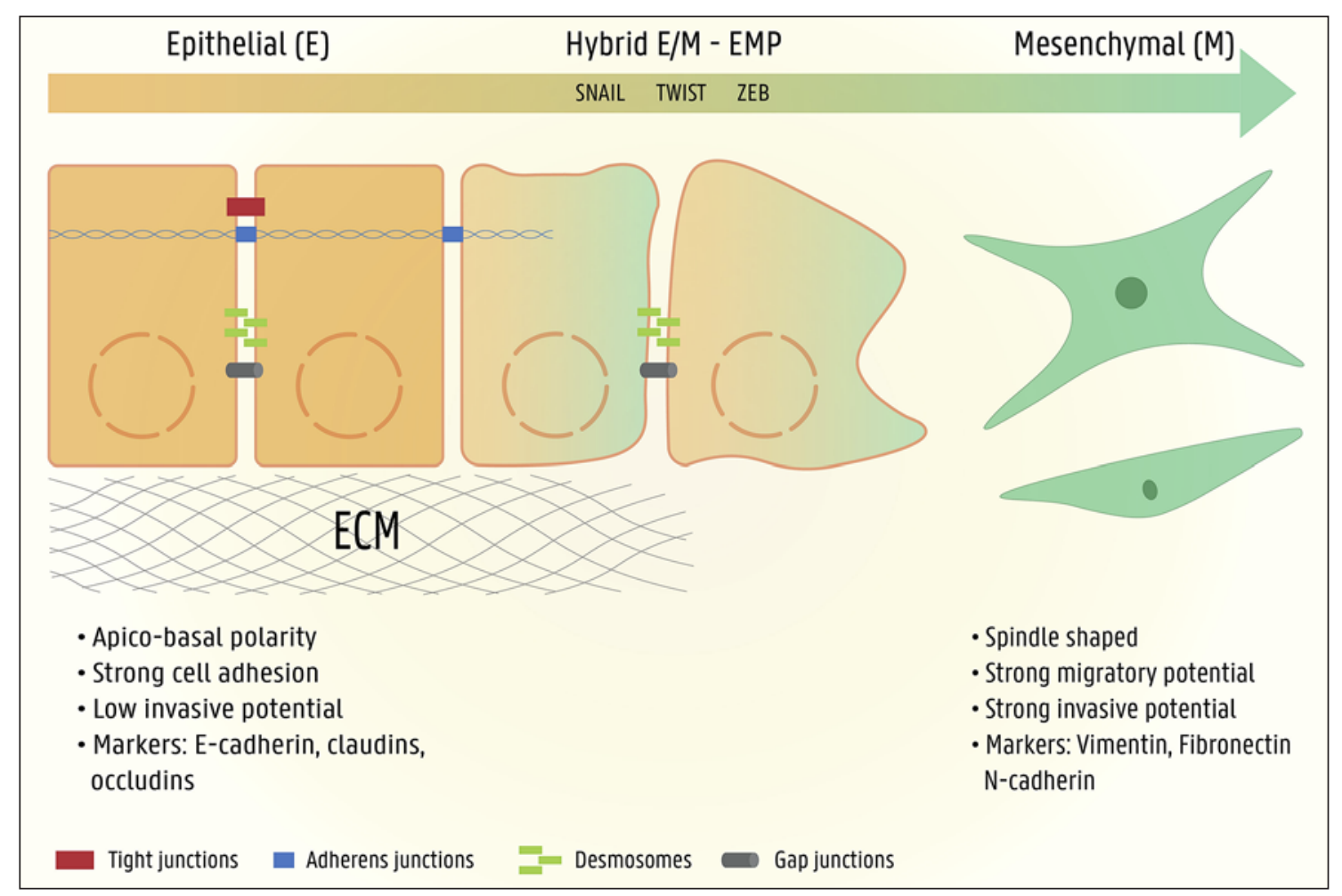

Fig. 1. Epithelial-mesenchymal transition (EMT). EMT encompasses a reversible, non-permanent phenotypical switch of wellpolarized epithelial cells to more spindle-shaped mesenchymallike cells with increased motile, migratory properties. Several phenotypic characteristics and epithelial and mesenchymal biomarkers that are used by researchers to document EMT in vitro and in vivo are listed. It is orchestrated by a network of EMT-associated transcription factors (EMT-TFs) of the SNAIL, TWIST, and ZEB fam- ily. As a result of partial EMT, a spectrum of hybrid E/M cells are often observed with both epithelial and mesenchymal traits. Cancer cells can reversibly switch between the continuum of hybrid states along the spectrum, allowing them to adapt easily and quickly to changing environmental cues. This ability is called epithelialmesenchymal plasticity (EMP). ECM, extracellular matrix; E, epithelial; M, mesenchymal.
2009]. Later, it was also found to play a role in adult organisms, under tight regulation in very specific conditions [Thiery et al., 2009; Stone et al., 2016]. For example, upon injury, EMT becomes activated to achieve re-epithelialisation and reorganisation of the extracellular matrix (ECM) during the process of wound healing.

When aberrantly activated, EMT can also contribute to pathologies such as fibrosis and cancer progression. Fibrosis is characterised by an excessive deposition of ECM by fibroblasts. The increased tissue scarring as a consequence of the ECM production leads to reduced organ function and eventually organ failure [Stone et al., 2016]. Another malignant condition in which EMT is aberrantly activated is cancer, where it has been demonstrated to play pivotal roles during metastasis. Induction of EMT in various cancer cell lines is associated with increased invasion potential in vitro and increased metastasis in vivo [Spaderna et al., 2006; Xu et al., 2017b]. In- versely, genetic loss of essential EMT drivers abrogated cancer cell plasticity, and several of these EMT drivers have been demonstrated to be essential for the metastatic spread in different murine cancer models [Yang et al., 2004; Krebs et al., 2017; Xu et al., 2017a].

The microenvironment plays important roles in the induction of the EMT programme. Several key signalling pathways, including transforming growth factor beta (TGF- $\beta$ ), Wnt, Notch, and Hedgehog [Wang et al., 2010b; Zhang et al., 2016], lead to the expression and regulation of a complex network of EMT-associated transcription factors (EMT-TFs) of the SNAIL, TWIST, and ZEB families [Cano et al., 2000; Comijn et al., 2001]. These EMTTFs directly repress a plethora of cell-cell adhesion genes essential for the strength and functionality of the epithelial barrier. At the same time, EMT-TFs directly or indirectly induce the expression of genes involved in cytoskeletal reorganisation, breakdown of the ECM, and survival. 
Fig. 2. Role of epithelial-mesenchymal transition (EMT)/mesenchymal-epithelial transition (MET) in the metastatic cascade of cancer progression. Schematic representation of the current model of involvement of EMT/MET in the metastatic cascade. Cancer cells at the primary tumour that undergo EMT acquire the ability to detach from the primary tumour mass either as single cells, or as small cell clusters. Once they have passed the basal membrane, they can invade in the surrounding tissue and gain access to a nearby located blood vessel (intravasation) to spread and disseminate. The obtained mesenchymal and stem cell properties allow the survival of the cancer cells in the blood stream. At a distant site in the body, interaction of the circulating tumour cells with the metastatic niche allows exit from the blood vessel (extravasation), drives MET, the reverse phenotypic switch of EMT, and allows the colonisation and outgrowth of a secondary tumour or metastasis.

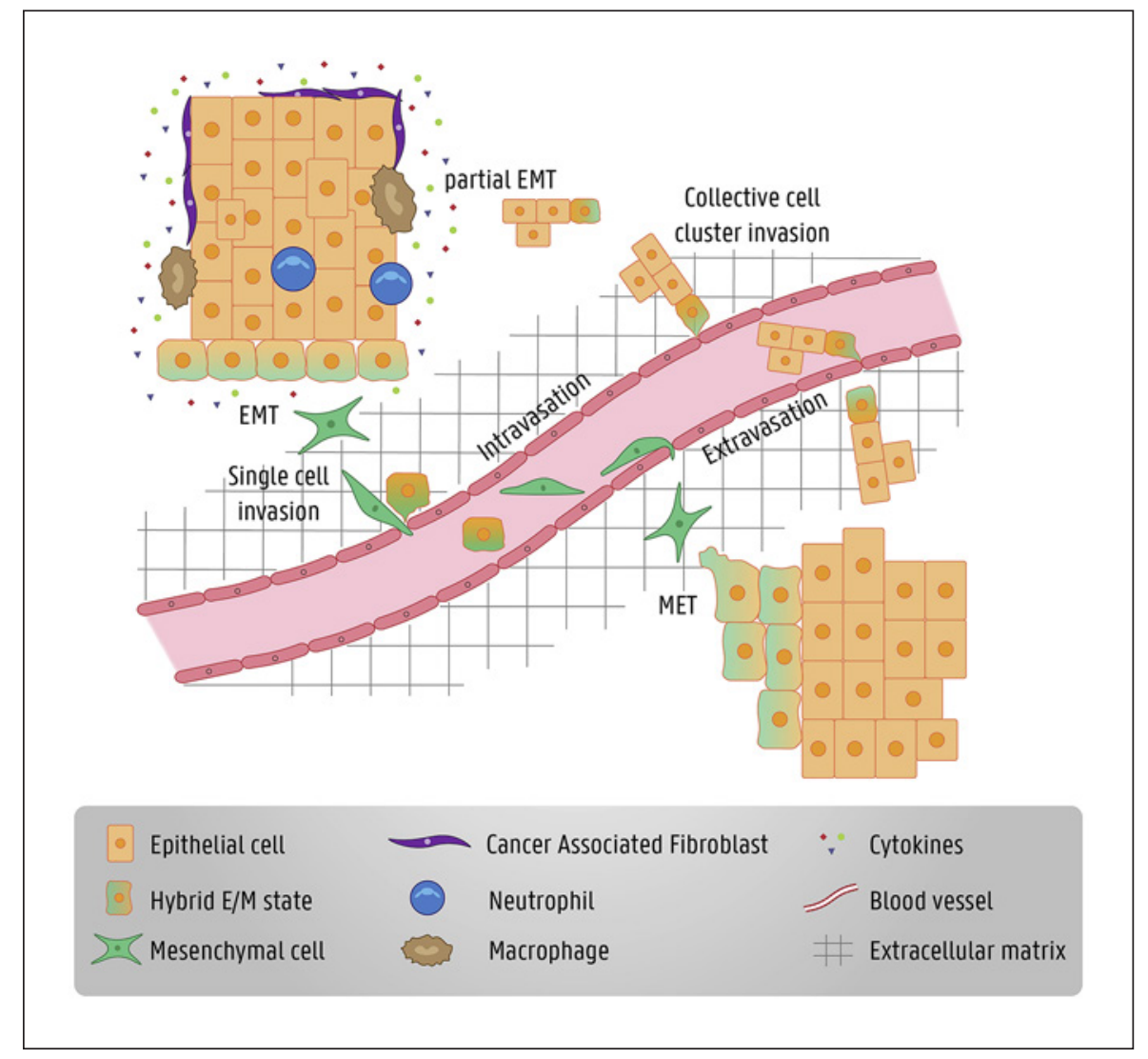

Combined, these transcriptional changes result in morphological changes that allow the cells to survive, invade, and migrate.

It is important to emphasize that cells undergoing EMT can acquire some mesenchymal characteristics while still maintaining epithelial ones, leading to so-called hybrid or intermediate EMT states (Fig. 1) [Chaffer et al., 2016; Nieto et al., 2016; Pastushenko et al., 2018; Godin et al., 2020]. Such partial EMT underlies the ability of cells to switch between a continuum of hybrid states along the epithelial-mesenchymal spectrum, which allows cells to easily adapt to environmental cues. This ability to adapt is termed epithelial-mesenchymal plasticity (EMP) [Ye and Weinberg, 2015; Nieto et al., 2016]. The high heterogeneity and plasticity of EMT gives tumour cells 2 routes for dissemination and hematogenous spread throughout the body. While fully mesenchymal cancer cells spread by single cell invasion, cancer cells that underwent a partial EMT, and have both epithelial and mesenchymal traits, adopt the ability to migrate collectively in cell clusters [Friedl and Alexander, 2011; Yu et al., 2013]. During collective migration, the leader cell at the front of the cluster underwent a partial EMT. Its mesenchymal traits enable the leader cell to guide migration and invasion, while its epithelial characteristics retain its adhesion with its neighbouring cancer cells and ensure the migration and invasion of the follower cells [Ye and Weinberg, 2015; Cheung and Ewald, 2016]. Collective migration of cell clusters dramatically increases the efficiency of seeding at distant sites [Pasquier et al., 2015]. In line with this, it was recently demonstrated that the hybrid cancer cells, as a result of partial EMT, have the most plasticity, and can be considered as the most malignant [Aiello et al., 2018; Pastushenko et al., 2018; Godin et al., 2020]. Fully reprogrammed mesenchymal cells appear to be locked in their state and have less potential to revert, which is essential for colonisation and the formation of distant nodules.

During metastatic dissemination, cancer cells undergo several steps before successfully seeding and secondary tumour formation. The metastatic cascade consists of 5 major steps: (i) invasion of the basement membrane and cell migration; (ii) intravasation into the surrounding vasculature or lymphatic system; (iii) survival in the circulation; (iv) extravasation from vasculature to secondary tissue; and finally (v) colonisation at secondary tumour sites (Fig. 2) [Massague and Obenauf, 2016; Hapach et al., 
2019]. Circulating tumour cells (CTCs), cancer cells which are present in the bloodstream, need to survive different types of stress. EMT induces and activates several survival pathways to resist anoikis or apoptosis (e.g., Akt, phosphatidylinositide-3-kinase [PI3K], epidermal growth factor receptor [EGFR]) [Cao et al., 2016; Gupta and Maitra, 2016]. Another way to increase the survival chances of the CTCs is by binding with platelets forming clusters creating an environment which protects the CTCs against shear stress, anoikis, and immune attacks [Haemmerle et al., 2018; Schlesinger, 2018]. The tissue factor is responsible for activating the platelets and inducing the coagulation cascade. Its expression is regulated by EMT as it is a target of several EMT-TFs [Bourcy et al., 2016; Genna et al., 2020]. CTCs also reprogram their metabolism to fulfil the continuous requirement of nutrient uptake during the metastatic cascade [Sciacovelli and Frezza, 2017]. Metabolic reprogramming frequently occurs in cancer cells to meet the increased demands for energy and biomolecule synthesis to support the increased proliferation. The best-known example is the Warburg effect, a switch from oxidative phosphorylation to glycolysis for ATP production. This also involves other metabolic pathways such as the TCA cycle and the lipid and amino acid metabolism. More and more evidence is gathering that the regulation of metabolic reprogramming is controlled by the EMT-TFs [Kang et al., 2019; Georgakopoulos-Soares et al., 2020].

In the context of cancer, EMT has also been correlated with the acquisition of cancer stem cell (CSC) properties [Mani et al., 2008; Morel et al., 2008]. CSCs or tumourinitiating cells are a subpopulation of cancer cells displaying characteristics of normal stem cells, such as the potential to self-renew, driving tumour growth, and the formation of secondary tumours [Yu et al., 2012; Ayob and Ramasamy, 2018]. The mesenchymal-like cells generated by EMT gain the ability to form spheroids, soft agar colonies, and upon transplantation form secondary tumours more efficiently. In addition, CSCs show increased resistance to chemotherapy due to their excessive drug efflux achieved by the increased expression of several cell membrane transporter proteins such as the ATP-binding cassette $(\mathrm{ABC})$ transporter protein family [Singh and Settleman, 2010; Du and Shim, 2016]. It is believed that these, often slow-cycling, therapy-resistant circulating CSCs may be the primary cause of relapsed disease. Next to the acquisition of CSC properties, cancer cells exploit another mechanism of therapy resistance, where EMT contributes to lineage identity switching [Boumahdi and de Sauvage, 2020]. The microenvironment forces the cancer cells to adopt a different cellular state by transdifferentiating into a cell lineage no longer depending on the survival pathway targeted by the therapy, allowing further growth.

\section{To Drug or Not to Drug EMT?}

As aberrant activation of EMT is involved in multiple pivotal steps during cancer progression (Fig. 2), it is no surprise that the pathways and molecular mechanisms orchestrating EMT are correlated with poor prognosis of cancer patients. As a consequence, increasing efforts are being made to search for opportunities to interfere with EMT in cancer cells and develop an anti-EMT adjuvant therapy [Davis et al., 2014].

It is expected that pharmacological targeting of EMT will lower the migratory and invasive behaviour of cancer cells at the primary tumour [Lin et al., 2018; Shen et al., 2019]. This would not only prevent the spread of cancer cells to distant sites throughout the body, it will also locally restrict the tumour to the primary site, resulting in more defined boundaries and making the resection of the primary tumour easier and more successful. This can only work on the condition that no early dissemination of the cancer cells has occurred. Several studies showed that dissemination from the primary tumour is occurring during the early stages of malignant progression [Nagrath et al., 2007; Husemann et al., 2008; Valastyan and Weinberg, 2011]. These early disseminated pre-neoplastic cells are able to survive in the circulation, extravasate, and survive at a new microenvironment [Husemann et al., 2008; Podsypanina et al., 2008]. They have the capacity to remain quiescent in a dormant state for a longer period or proliferate to immediately form metastases at the distant organ site, prior to detection of the cancer [Podsypanina et al., 2008; Friberg and Nyström, 2015]. In a later disease stage, EMT-inhibitors could be applied to prevent the colonisation of the tumour of origin by CTCs, a process called tumour self-seeding [Kim et al., 2009]. This process has a higher efficiency than the formation of secondary metastases as the neovasculature is leaky at the primary tumour, and there is no need to adapt to the new microenvironment [Carmeliet and Jain, 2000]. Another expected consequence of interfering with EMT is a drop in the acquisition of CSC properties [Bao et al., 2011; Bogachek et al., 2014; Mladinich et al., 2016], which would reduce the ability of CTCs to initiate secondary tumours when they seed at distant sites. In addition, if the cancer cells no longer acquire CSC properties, they most probably will 
not gain expression of $\mathrm{ABC}$ transporters and no longer have an increased drug efflux [Singh and Settleman, 2010; Du and Shim, 2016], which would increase the efficacy of classical chemotherapy [Meidhof et al., 2015; Zhou et al., 2015]. Furthermore, it is expected that reducing the population of slow-cycling, therapy-resistant CTCs will significantly reduce the risk of relapse. In conclusion, therapeutic targeting of EMT would prevent the formation of metastases by reducing the invasion and dissemination of cancer cells. If this is combined with the increased vulnerability to the classical anti-cancer chemotherapies, the primary tumour would be much more treatable, leading to better patient outcomes, with less chance of recurrence.

However, on the other side of the coin, some criticisms and putative problems have been raised regarding the use of anti-EMT therapies. Recently, there were critical comments about the necessity of EMT and the expression of EMT-TFs in the metastatic cascade [Ye et al., 2017; Williams et al., 2019]. These were based on a study whereby the genetic inactivation of 2 independent EMT-TFs, Snail and Twist, had no effect on the metastatic potential of mouse breast and pancreatic adenocarcinoma, but solely affected the chemoresistance [Fischer et al., 2015; Zheng et al., 2015]. This was counteracted by demonstrating that another EMT-TF, Zeb1, was essential for the metastatic potential and chemoresistance in the same pancreatic cancer mouse model [Krebs et al., 2017]. As such, with this study, clear-cut genetic evidence was provided that targeting the molecular drivers of EMT could efficiently target multiple aspects of tumourigenesis and could have a beneficial impact for the outcome of cancer patients.

A major putative risk with an anti-EMT strategy is that it could drive the reverse process, mesenchymal-epithelial transition (MET), in CTCs and favour colonisation of early-disseminated cells from the primary tumour. Additionally, while EMT is associated with many pathogenic consequences such as an increase in migration and invasion, it is also associated with some beneficial features such as a decreased proliferation [Salt et al., 2014]. Consequently, treating cancer patients with drugs that intervene with EMT will likely not only lead to MET and reepithelialisation, but also to increased tumour proliferation [Pasquier et al., 2015]. It could not only cause a faster growth of the primary tumour, but may also initiate the outgrowth of putative secondary quiescent micrometastases. On the other hand, this would make the cancer cells more susceptible to classical chemotherapeutics, that mainly target fast proliferating cells. It is of the uttermost importance that sufficient research is focussing on the balance between increased proliferation and chemotherapy susceptibility upon an anti-EMT strategy which drives MET. If the balance shifts towards more proliferation without increasing the efficacy of chemotherapeutics, we risk stimulating the progression and growth without improving the patients' prognosis [Pattabiraman et al., 2016].

These concerns raise important questions. Which patients would benefit the most from an anti-EMT therapy: patients with early stage disease, or late stage with detectable CTCs and evidence of distant metastases? In each of these phases during metastatic progression, EMT plays different transient roles which are highly dependent on the cellular state and microenvironment cues [Pasquier et al., 2015]. As such it can be expected that within one patient an anti-EMT therapy may be beneficial for some cancer cell subpopulations, but may have adverse effects on others. Second, it is obvious that an anti-EMT therapy will need to be utilised in conjunction with already established anti-tumour drugs [Singh et al., 2018], but which drugs, how and when? More preclinical research is necessary to answer these important questions.

We also need to consider whether EMT-targeted therapy could potentially lead to serious side-effects. As discussed, EMT is also important in the physiological response to wounding and the process of wound healing [Friedl and Alexander, 2011; Yu et al., 2013]. It can be expected that wound healing would be impaired and the strategy of targeting EMT should be carefully designed. But there may be more and other potential side-effects. Many of the molecular drivers, the EMT-TFs, have been proven to have other non-EMT functions including normal adult stem cell regulation and differentiation, tissue homeostasis, and immune responses. Therefore, it is of the utmost importance that we keep on studying EMT and its molecular regulation both under normal development and physiological conditions, and upon malignant transformation. Only in this way we will find potential therapeutic targets with reduced chance of side-effects.

Four different strategies have been proposed so far to target EMT: (i) preventing EMT by blocking the upstream extracellular signals and their signal transduction pathways; (ii) inhibiting the expression or functions of critical molecular drivers, the EMT-TFs; (iii) targeting the mesenchymal cells and their interaction with the ECM or (iv) inhibition of MET (Fig. 3) [Davis et al., 2014]. Over recent decades, most progress has been made when inhibiting the upstream paracrine pathways and targeting the expression or functions of EMT-TFs and their co-factors. 


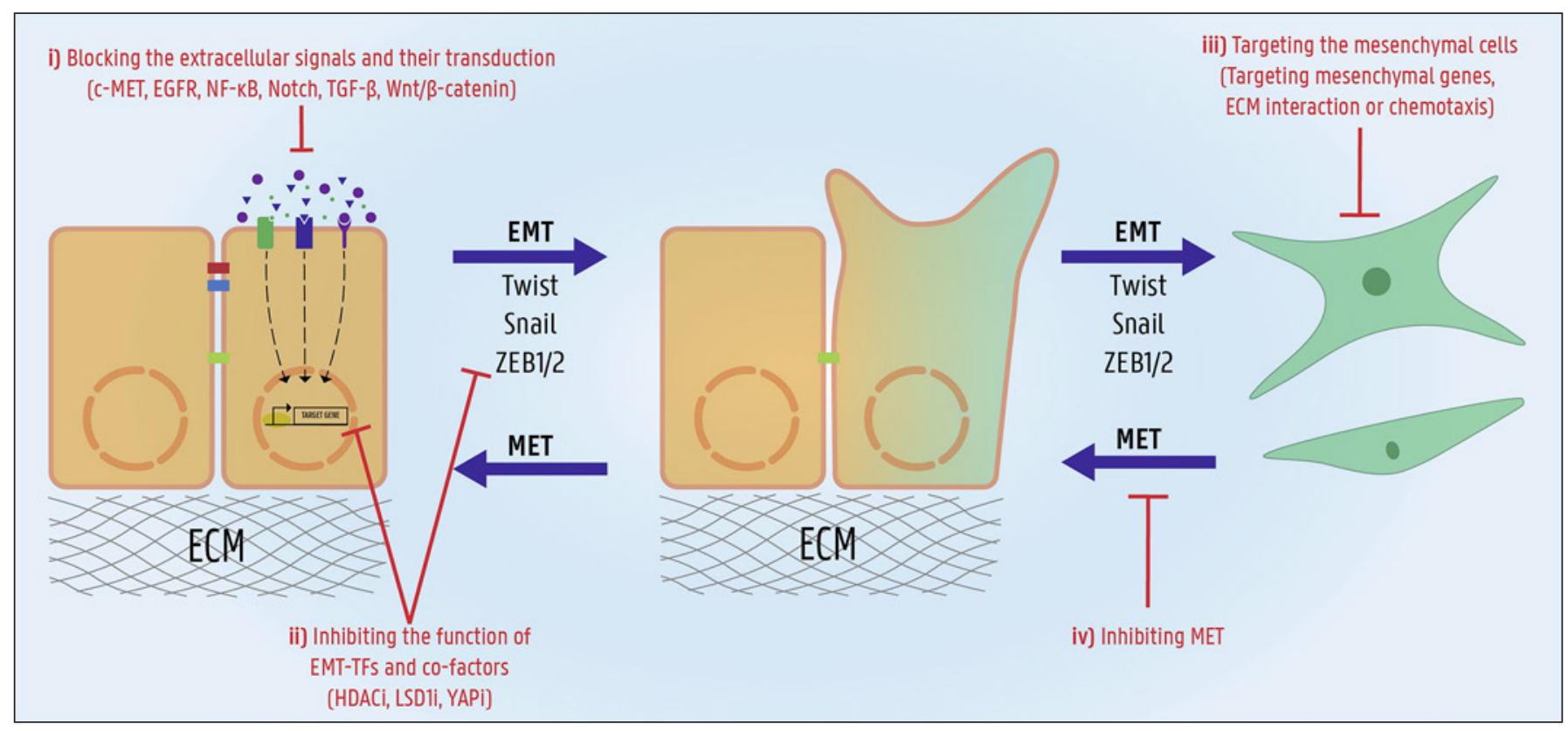

Fig. 3. Potential therapeutic strategies to interfere with epithelial-mesenchymal transition (EMT). Four potential strategies have been proposed to therapeutically interfere with EMT in cancer to reduce the metastatic burden: i) preventing EMT by blocking the extracellular paracrine EMT-inducing factors and their signalling pathways, or ii) disrupting the expression or function of EMT-associated transcription factors (EMT-TFs) and their essential co-factors, iii) targeting the mesenchymal cells, and iv) inhibition of mesenchymal-epithelial transition (MET).

\section{Anti-EMT Targeting Strategies: Blocking Upstream Signalling Pathways}

EMT plays crucial roles during embryonic development; therefore it is not a surprise that our knowledge regarding upstream paracrine signals predominantly originates from developmental studies. As expected, multiple developmental signalling pathways, like the $\mathrm{Wnt}$, Notch, and bone morphogenetic protein (BMP)/TGF- $\beta$ pathways, which are involved in body plan organization of the embryo, also affect EMT in a cancer setting.

The best-known inducer of EMT is TGF- $\beta$ [Thiery et al., 2009; Fabregat et al., 2014; Lamouille et al., 2014; Zarzynska, 2014], a multifunctional cytokine belonging to a larger family of structurally and functionally related proteins including activins/inhibins and the BMPs [Derynck and Budi, 2019]. This family of proteins regulates many different cellular functions such as proliferation, apoptosis, differentiation, EMT, and migration/invasion [Fabregat et al., 2014; Syed, 2016]. Binding of the ligand to the TGF- $\beta$ receptor (TGF- $\beta R$ ) results in phosphorylation and activation of the receptor SMADs (R-SMADs), which can bind the co-SMAD. R-SMAD/co-SMAD complexes translocate to the nucleus and induce the transcription of the TGF- $\beta$ target genes, including the EMT-TFs [Hata and Chen, 2016]. Although TGF- $\beta$ is a well-known inducer of EMT in vitro, it has a dual role in cancer progression in vivo, known as the TGF- $\beta$ paradox [Bachman and Park, 2005; Bierie and Moses, 2010]. In the early stages of cancer initiation and progression, this cytokine acts as a potent anti-cancer agent by inhibiting the cell cycle progression of epithelial cells and triggering apoptosis. However, during cancer progression, genetic and epigenetic changes conspire to revert TGF- $\beta$ to a promoter of angiogenesis, growth, invasion, metastasis, and therapy resistance. At what point the role of TGF- $\beta$ switches from tumour suppressor to oncogene still remains poorly understood. Since TGF- $\beta$ is implicated in a broad spectrum of cancer-promoting effects, it is a popular target for potential clinical therapies with multiple anti-TGF- $\beta$ inhibitors currently tested in several clinical studies (Table 1) [Zhang et al., 2016]. The approaches used to therapeutically target TGF- $\beta$ can be classified into 3 types: (i) antisense oligonucleotides, (ii) ligand traps, and (iii) small molecules used to inhibit the intracellular signalling cascade. The antisense oligonucleotides are single-strand nucleotide 
Table 1. Overview of clinical trials testing TGF- $\beta$ inhibitors as anti-cancer therapy

\begin{tabular}{|c|c|c|c|c|}
\hline Drug & Target & $\begin{array}{l}\text { Development } \\
\text { stage }\end{array}$ & Cancer type & Trial identifier \\
\hline Galunisertib monohydrate & TGF- $\beta$ R1 & Phase II & (Metastatic) breast cancer & NCT02538471 (T) \\
\hline LY-3022859 & TGF- $\beta$ R2 & Phase I & Solid cancers & NCT01646203 (T) \\
\hline \multirow[t]{2}{*}{ SHR-1701 } & TGF- $\beta$ R2 & Phase I & Solid cancers & NCT04324814(O) \\
\hline & & & & NCT03710265 (O) \\
\hline YL-13027 & TGF- $\beta$ R1 & Phase I & Solid cancers & NCT03869632 (O) \\
\hline
\end{tabular}

Clinical trials were searched on the Pharma Intelligence Centre GlobalData database by performing a clinical trial search with TGF- $\beta$ as target in the oncology therapy area and application of the drug as monotherapy; only the most advanced clinical trials are shown. $\mathrm{CN}$, completed (no results available); O, ongoing; P, planned; T, terminated; TGF- $\beta$ R, transforming growth factor beta receptor.

molecules (13-25 nucleotides) which prevent protein expression by directing their target mRNA (in this case encoding a gene involved in TGF- $\beta$ synthesis) for mRNA degradation [Bennett and Swayze, 2010]. The ligand traps are ligand neutralising antibodies and soluble decoy receptors binding to and trapping the TGF- $\beta$ ligands, preventing their interaction with and activation of the TGF$\beta$ Rs. The last therapeutic strategy is the small molecules which can either block the TGF- $\beta$ R activity by acting as an ATP-competitive inhibitor for the kinase catalytic enzymatic site or interfere with the function of the TGF- $\beta$ intracellular transducers, the SMADs. The pleiotropic roles of TGF- $\beta$ in cancer development and progression, but also its essential role in embryonic development and normal cell physiology, make it a tricky target for anticancer therapies. It is not recommended to have a systemic blockage of the TGF- $\beta$ pathway, but it should be used in a cancer-specific context with as much anti-cancer specificity as possible to reduce the potential side-effects [Connolly et al., 2012; Sim et al., 2019].

Next to the canonical SMAD-dependent downstream signalling, the TGF- $\beta$ R complex is also able to transmit signals through other factors, including the mitogen-activated protein kinases (MAPKs), PI3K, tumour necrosis factor (TNF) receptor-associated factor 4/6 (TRAF4/6), and Rho family of small GTPases [Costanza et al., 2017]. This transverse non-canonical signalling allows TGF- $\beta$ to exert its pleiotropic functions and to integrate signals from several different cellular processes such as the cell cycle or apoptosis. TGF- $\beta$ non-canonical signalling also mediates EMT, although this part is much less studied [Syed, 2016].
Numerous feedback mechanisms within the TGF- $\beta$ pathway exist, and a versatile crosstalk with other signalling pathways has been described, including multiple receptor tyrosine kinases (RTKs) like the EGFR, and c-Met [Misra et al., 2012; Kim et al., 2016; Zhang Y et al., 2018]. Upon activation of these receptors, the ligand induces receptor dimerization leading to phosphorylation of specific tyrosine residues in the intracellular domain, recruiting downstream activators [Seshacharyulu et al., 2012]. Both receptors are frequently amplified or mutated in many different carcinoma types leading to over-activation of the downstream signalling pathway and contributing to tumour progression by increasing cancer cell proliferation, promoting invasion via upregulation or activation of matrix metalloproteinases (MMPs) [Normanno et al., 2006; Rajaram et al., 2017], and resistance to targeted therapies [Zhang Y et al., 2018]. Many pre-clinical and clinical studies are being performed with inhibitors against EGFR or c-Met (Table 2) demonstrating inhibition of EMT and decreased tumour progression. Downstream, activated RTKs recruit a wide variety of proteins with Src homology-2 (SH2) or phosphotyrosinebinding (PTB) domains, resulting in activation of multiple signal transduction cascades, including the RAS/ MAPK, PI3K/AKT/mTOR, and JAK/STAT pathways. There are multiple commercially available inhibitors targeting these pathways that have been tested both in vitro and in vivo [Tian T et al., 2019; Yang et al., 2019; Verhoeven et al., 2020]. However, their ability to suppress EMT is highly dependent on the cellular context and mutational status of the cancer cells. 
Table 2. Overview of clinical trials testing receptor tyrosine kinase EGFR and c-MET inhibitors as anti-cancer therapy

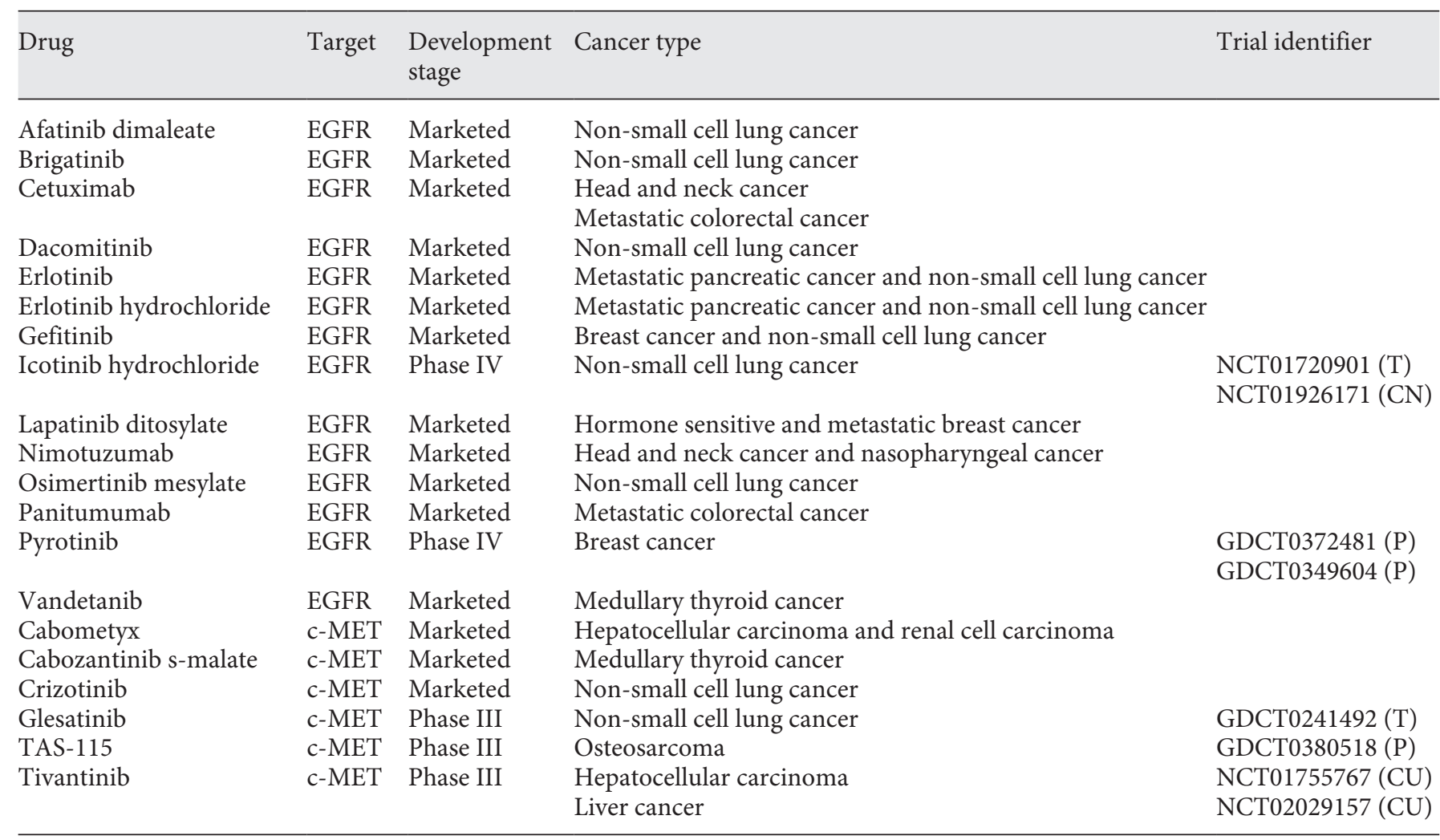

Clinical trials were searched on the Pharma Intelligence Centre GlobalData database by performing a clinical trial search with EGFR and c-MET as target in the oncology therapy area and application of the drug as monotherapy. EGFR inhibitors mentioned are tested in at least 5 different clinical trials, but only the most advanced clinical trials are shown. The marketed status was defined by performing an advanced drug search on the same database for the same targets. CN, completed (no results available); CU, completed unsuccessfully; EGFR, epidermal growth factor receptor; P, planned; T, terminated.

Another extremely versatile pathway able to induce EMT is the Wnt signalling pathway [Thiery et al., 2009; Basu et al., 2018]. The regulation of cell proliferation and differentiation by the Wnt signals is critical during the early stages of embryonic development [Komiya and Habas, 2008]. It is a very complex pathway as it consists of 19 different glycolipoprotein Wnt ligands, different types of receptors (Frizzled, RTK, and LRP5/6), and many different combinations of ligand-receptor interactions [Miller, 2002; MacDonald and $\mathrm{He}, 2012$ ]. In addition to these ligands activating the receptors, there are also a number of secreted antagonists competing for the receptors and reducing the signalling activity [Cruciat and Niehrs, 2013]. On top of this, there is a lot of crosstalk with other signalling pathways including RTKs and TGF- $\beta$ [Patel et al., 2019]. Downstream, the receptors can use $\beta$-catenin-dependent (canonical) and/or $\beta$-catenin- independent (non-canonical) signal transduction pathways [Niehrs, 2012]. In the canonical pathway, $\beta$-catenin is targeted for proteasomal degradation by a "destruction complex" only in the absence of the Wnt ligands. Activation of the Wnt receptors prevents the phosphorylation of $\beta$-catenin by GSK3 $\beta$ and allows it to accumulate in the cytoplasm and translocate to the nucleus [Shaw et al., 2019]. There, $\beta$-catenin interacts with TCF and LEF transcription factors, and together they control essential genes for cellular self-renewal and proliferation. The noncanonical pathways, such as the PCP and $\mathrm{Ca}^{2+}$ pathways, do not rely on $\beta$-catenin, but use the small GTPase and JUN-N terminal kinases or the phospholipase $\mathrm{C}$ to control cytoskeletal changes, cell survival, migration, and invasion, respectively [Weeraratna et al., 2002; De, 2011]. In cancer, uncontrolled activation of Wnt signalling contributes to increased proliferation and acquisition and 
Table 3. Overview of clinical trials testing Wnt and $\beta$-catenin inhibitors as anti-cancer therapy

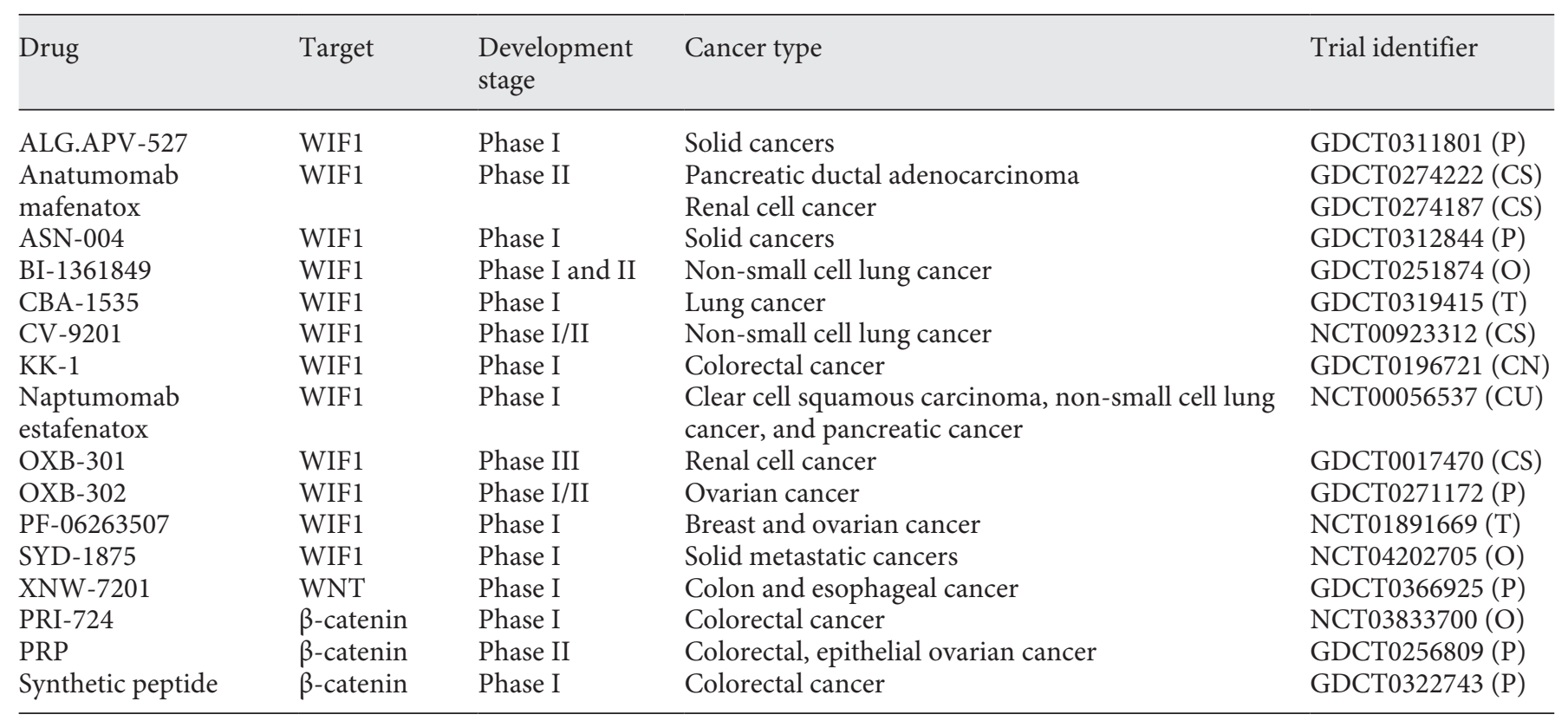

Clinical trials were searched on the Pharma Intelligence Centre GlobalData database by performing a clinical trial search with Wnt and $\beta$-catenin as target in the oncology therapy area and application of the drug as monotherapy. Only the most advanced clinical trials are shown. CN, completed (no results available); CS, completed successfully; CU, completed unsuccessfully; O, ongoing; P, planned; T, terminated; WIF1, Wnt activated inhibitory factor 1 .

propagation of CSCs, which contributes to chemoresistance and also dampens the anti-tumor immune response in the tumour microenvironment [Patel et al., 2019]. The Wnt signalling pathway regulates EMT as the interaction of $\beta$-catenin with the LEF transcription factors downregulates the cell adhesion genes, leading to induction of EMT, increased invasion, and metastasis [Heuberger and Birchmeier, 2010; Hu et al., 2019]. As the Wnt pathway is often seen to be required for cancer cell survival, it has often been suggested as a valid therapeutic target. However, the biggest challenge is to find therapeutic strategies that specifically target the cancer cells without hampering its essential role for non-malignant cells. Several therapies targeting this versatile pathway are currently being tested in pre-clinical and clinical studies (Table 3), and there have been some promising results.

The Notch signalling pathway is a much less complex transduction pathway, but with very diverse effects based on the signal dose and the cellular context [Kopan, 2012]. The interaction of the Notch ligands, Delta-like and Jagged, with Notch receptors on neighbouring cells, causes a cleavage of the transmembrane receptor, resulting in the release of the Notch intracellular domain allowing trans- location to the nucleus [Bray, 2006; Miele, 2006]. ADAM17 and $\gamma$-secretase proteases are responsible for the ectodomain shedding and release of the Notch intracellular domain from the inner side of the cell membrane [Mumm et al., 2000; Reiss and Saftig, 2009]. Activation of this pathway promotes cancer cell growth and regulates the cancer-related neoangiogenesis. Next to the involvement of Notch signalling in these malignant cancer characteristics, it also positively regulates the expression of EMT-TFs, increasing the metastatic burden [Zhang J et al., 2018; Kar et al., 2019]. However, these effects are strongly dependent on the cellular context [Palomero et al., 2006; Wang et al., 2010a; Kuhnert et al., 2011; Capaccione and Pine, 2013; Olsauskas-Kuprys et al., 2013; Sonoshita et al., 2015], which limits the applicability of Notch and $\gamma$-secretase inhibitors (Table 4) to specific cancer/subtype situations, with only specific patient profiles benefiting from this treatment.

Finally, multiple pro-inflammatory signals have been demonstrated to be potent inducers of EMT, including TNF- $\alpha$. One of the pathways regulated by TNF- $\alpha$ is based on the nuclear factor kappa-B (NF- $\kappa \mathrm{B})$ family of transcription factors, which are a family of 5 subunits forming 
Table 4. Overview of clinical trials testing Notch and $\gamma$-secretase inhibitors as anti-cancer therapy

\begin{tabular}{|c|c|c|c|c|}
\hline Drug & Target & $\begin{array}{l}\text { Development } \\
\text { stage }\end{array}$ & Cancer type & Trial identifier \\
\hline \multirow[t]{2}{*}{ Tarextumab } & \multirow[t]{2}{*}{ NOTCH2 } & \multirow[t]{2}{*}{ Phase I/II } & Pancreatic cancer & NCT01647828 (CS) \\
\hline & & & Small cell lung cancer & NCT01859741 (T) \\
\hline AL-101 & $\gamma$-secretase & Phase II & Triple-negative breast cancer & GDCT0368476 (P) \\
\hline AL-102 & $\gamma$-secretase & Phase I & Basal cell carcinoma & NCT01986218 (T) \\
\hline LY-900009 & $\gamma$-secretase & Phase I & Advanced cancers & NCT01158404 (CS) \\
\hline MK-0752 & $\gamma$-secretase & Phase I & Metastatic breast cancer & NCT00106145 (CS) \\
\hline Nirogacestat hydrobromide & $\gamma$-secretase & Phase III & Soft tissue sarcoma & GDCT0313060 (O) \\
\hline \multirow[t]{2}{*}{ RG-4733 } & \multirow[t]{2}{*}{$\gamma$-secretase } & \multirow[t]{2}{*}{ Phase II } & Metastatic, triple-negative breast cancer & NCT01151449 (T) \\
\hline & & & Colorectal cancer & NCT01116687 (CU) \\
\hline Tarenflurbil & $\gamma$-secretase & Phase II & Prostate cancer & NCT00045123 (CN) \\
\hline
\end{tabular}

Clinical trials were searched on the Pharma Intelligence Centre GlobalData database by performing a clinical trial search with Notch and $\gamma$-secretase as target in the oncology therapy area and application of the drug as monotherapy. Only the most advanced clinical trials are shown. CN, completed (no results available); CS, completed successfully; CU, completed unsuccessfully; O, ongoing; P, planned; T, terminated.

Table 5. Overview of clinical trials testing NF- $\kappa B$ inhibitors as anti-cancer therapy

\begin{tabular}{|c|c|c|c|c|}
\hline Drug & Target & $\begin{array}{l}\text { Development } \\
\text { stage }\end{array}$ & Cancer type & Trial identifier \\
\hline ACT-001 & $N F-\kappa B$ & Phase I/II & Brain cancer & GDCT0384012 (P) \\
\hline CIGB-552 & $\mathrm{NF}-\kappa \mathrm{B}$ & Phase I & Solid cancers & GDCT0239987 (T) \\
\hline \multirow[t]{2}{*}{ Curcumin } & $N F-\kappa B$ & Phase II/III & Head and neck cancer & GDCT0016710 (CN) \\
\hline & & Phase III & Cervical cancer & GDCT0120361 (T) \\
\hline ETS-2101 & $N F-\kappa B$ & Phase II & Brain cancer and glioma & GDCT0184220 (T) \\
\hline LH-025 & $N F-\kappa B$ & Phase I & Solid cancers & GDCT0253114 (CN) \\
\hline \multirow[t]{2}{*}{ OMN-54 } & $N F-\kappa B$ & Phase I/II & Colorectal and ovarian cancer & NCT01555242 (CS) \\
\hline & & Phase II & Prostate cancer & NCT03495479 (P) \\
\hline Teglarinad chloride & $N F-\kappa B$ & Phase I & Lymphoma and solid cancers & NCT00457574 (T) \\
\hline UNBS-1450 & $N F-\kappa B$ & Phase I & Lymphoma and solid cancers & GDCT0269013 (CN) \\
\hline
\end{tabular}

Clinical trials were searched on the Pharma Intelligence Centre GlobalData database by performing a clinical trial search with NF$\kappa \mathrm{B}$ as target in the oncology therapy area and application of the drug as monotherapy. Only the most advanced clinical trials are shown. $\mathrm{CN}$, completed (no results available); CS, completed successfully; NF- $\mathrm{B}$, nuclear factor kappa-B; O, ongoing; $\mathrm{P}$, planned; T, terminated.

a functional transcription factor by dimerization [Xia et al., 2018]. Upon activation of TNF- $\alpha$, NF- $\kappa B$ translocates to the nucleus to drive expression of downstream effectors, eventually inducing the expression and protein stabilization of multiple EMT-TFs [Helbig et al., 2003; Maier et al., 2010]. As such, the NF- $\kappa$ B pathway not only plays a critical role by protecting the transformed cells from apoptosis [Xia et al., 2014; Xia et al., 2018], but also promotes EMT leading to increased invasiveness of many different cancer types [Helbig et al., 2003; Maier et al., 2010]. Therapeutic targeting of the NF- $\kappa$ B pathway (Table 5) might lead to inhibition of EMT and reduced metastatic burden in combination with regained ability to undergo apoptosis. 
Table 6. Overview of clinical trials testing HDAC1/2 inhibitors as anti-cancer therapy

\begin{tabular}{|c|c|c|c|c|}
\hline Drug & Target & $\begin{array}{l}\text { Development } \\
\text { stage }\end{array}$ & Cancer type & Trial identifier \\
\hline Domatinostat & $\mathrm{HDAC} 1 / 2 / 3$ & Phase II & Small cell lung cancer & GDCT0245900 (T) \\
\hline Entinostat & $\mathrm{HDAC} 1 / 3$ & Phase II & $\begin{array}{l}\text { Breast cancer } \\
\text { Metastatic melanoma }\end{array}$ & $\begin{array}{l}\text { NCT00828854 (CS) } \\
\text { NCT00185302 (CS) }\end{array}$ \\
\hline Fimepinostat & $\mathrm{HDAC} 1 / 2 / 3 / 6 / 10$ & Phase II & Thyroid cancer & NCT03002623 (T) \\
\hline Mocetinostat & $\mathrm{HDAC} 1 / 4$ & Phase II/III & Bladder cancer & NCT02236195 (CU) \\
\hline Pracinostat & $\mathrm{HDAC} 1 / 2 / 4$ & Phase II & Prostate cancer & NCT01075308 (CS) \\
\hline Remetinostat & $\mathrm{HDAC} 1 / 2 / 3 / 6$ & Phase II & Basal cell carcinoma & NCT03180528 (O) \\
\hline Resminostat & $\mathrm{HDAC} 1 / 2 / 4$ & Phase III & Hepatocellular carcinoma & $\begin{array}{l}\text { GDCT0168772 (T) } \\
\text { GDCT0216169 (T) }\end{array}$ \\
\hline Tucidinostat & $\mathrm{HDAC} 1 / 2 / 3 / 8 / 10$ & Marketed & Metastatic breast cancer & \\
\hline Vorinostat & $\mathrm{HDAC} 1 / 2 / 3 / 6$ & Phase III & Lung cancer & NCT00419367 (CN) \\
\hline
\end{tabular}

Clinical trials were searched on the Pharma Intelligence Centre GlobalData database by performing a clinical trial search with $\mathrm{HDAC} 1 / 2$ as target in the oncology therapy area and application of the drug as monotherapy. Only the most advanced clinical trials are shown. The marketed status was defined by performing an advanced drug search on the same database for the same targets. $\mathrm{CN}$, completed (no results available); CS, completed successfully; CU, completed unsuccessfully; HDAC, histone deacetylase; O, ongoing; T, terminated.

\section{Anti-EMT Targeting Strategies: Targeting the Molecular Drivers of EMT}

EMT is orchestrated on the transcriptional level by the upregulation of a network of EMT-TFs, including members of the SNAIL, TWIST, and ZEB families, that directly repress epithelial genes and upregulate mesenchymal genes. Conditional loss-of-function mouse models have demonstrated that such EMT-TFs are truly important and indispensable drivers/regulators of the EMT programme in the context of cancer progression [Yang et al., 2004; Krebs et al., 2017; Xu et al., 2017a]. Therefore, one can assume that perturbing the expression and/or the functions of these EMT-TFs would be one of the most effective manners to perturb EMT, but this is technically very challenging. The expression levels of the different EMT-TFs are tightly entangled via multiple feedback mechanisms. In addition, several EMT-TFs have complementary and redundant functions, with the specific role of a particular EMT-TF highly depending on the cellular context and microenvironment. As such, targeting EMTTFs is likely not the most ideal therapeutic solution, rather, targeting their interactions with essential co-factors might be a more beneficial strategy. In this way it would be possible to target multiple EMT-TFs at the same time, as they often share the same co-factors. This could also reduce putative side-effects by targeting only cancer-specific co-factors and sparing the roles of EMT-TFs under normal physiological conditions.
The Yes-associated protein (YAP) was identified as a co-factor of the EMT-TF ZEB1. YAP lacks the presence of a DNA binding site and requires the interaction with members of the TEAD family or other families of transcription factors to exert its function. The activity of YAP is controlled by the large tumour suppressor (LATS) kinases of the Hippo pathway, targeting YAP for cytoplasmic retention and ubiquitin-mediated protein degradation. YAP activation or overexpression results in induction of EMT in different cancer types [Yuan et al., 2016; Ling et al., 2017; Kim and Myung, 2018]. The recently identified interaction of YAP with ZEB1 turns this EMTTF into a transcriptional activator, and results in increased metastatic burden [Lehmann et al., 2016], making it an interesting therapeutic target. Several preclinical studies have been performed to identify and study the effects of molecules perturbing this interaction, with verteporfin being the first tested in phase II clinical trials for treating metastatic breast cancer and advanced stages of pancreatic cancer.

Targeting epigenetic modulation during EMT has gained increasing attention over the last couple of years as this modulation is seen as a reversible, stable way to induce the reprogramming required for the phenotypic switch [Skrypek et al., 2017]. The epigenetic regulation during EMT is coordinated by the interaction of the EMT-TFs with different chromatin remodelling complexes like the NuRD complex, the SIN3A repressor complex, the SWI/SNF complex, and the PRC2 complex, all 
Table 7. Overview of clinical trials testing LSD1 inhibitors as anti-cancer therapy

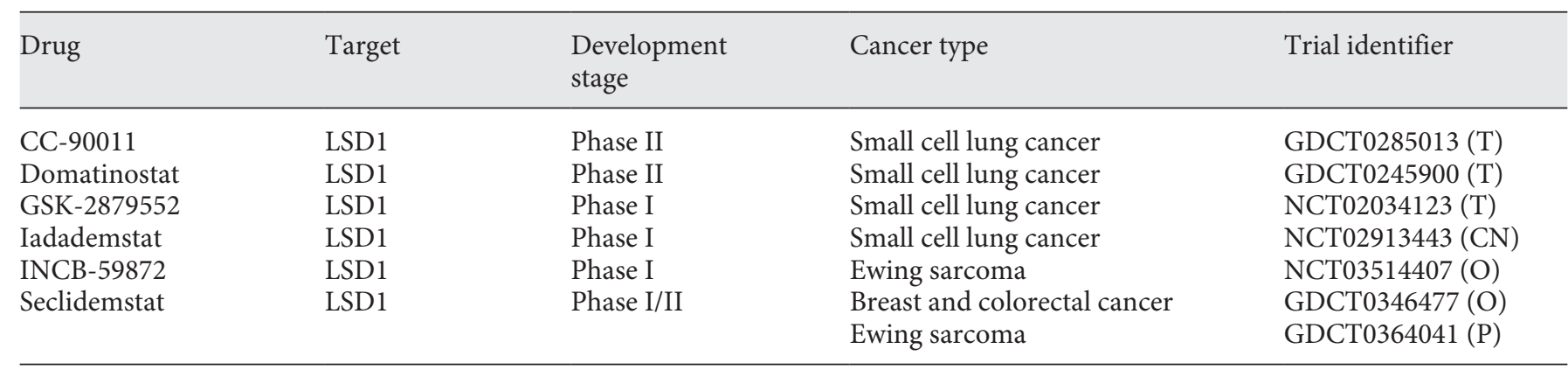

Clinical trials were searched on the Pharma Intelligence Centre GlobalData database by performing a clinical trial search with LSD1 as target in the oncology therapy area and application of the drug as monotherapy. Only the most advanced clinical trials are shown. $\mathrm{CN}$, completed (no results available); LSD1, lysine-specific histone demethylase 1; O, ongoing; P, planned; T, terminated.

involved in histone (de)acetylation and (de)methylation [Verstappen et al., 2008; Fukagawa et al., 2015; Skrypek et al., 2017]. Histone deacetylases (HDACs) are one class of these epigenetic modulators for which inhibitors are available. As part of the aforementioned chromatin remodelling complexes, HDACs are recruited to the promoter of epithelial genes, leading to local $\mathrm{H} 3$ and $\mathrm{H} 4$ deacetylation and subsequent silencing [ $\mathrm{Li}$ and Seto, 2016]. HDAC inhibitors (Table 6) are able to revert the acetylation status, thereby reactivating the epithelial gene expression. However, while the inhibition of HDACs could repress EMT and tumour growth in some models, other models showed the opposite with an induction of EMT-TF expression leading to enhanced EMT [Jiang et al., 2013; Ji et al., 2015]. An alternative for the broad-spectrum drugs that target the enzymatic capacity of these cofactors would be to specifically disrupt the interactions between EMT-TFs and the chromatin remodelling complexes.

Another epigenetic regulator that binds SNAIL and ZEB EMT-TFs is the lysine-specific histone demethylase 1 (LSD1), a protein that demethylates H3K4 and H3K9. Similar as demonstrated for the HDACs, LSD1 is recruited by the EMT-TFs to their target promoters, resulting in an altered epigenetic landscape, allowing EMT to occur [McDonald et al., 2011; Skrypek et al., 2017; Boulding et al., 2018]. LSD1 expression was correlated with poor patient survival in many different types of cancer, but its function varies significantly depending on the tumour type [Maiques-Diaz and Somervaille, 2016; Majello et al., 2019]. Several pre-clinical studies have shown that LSD1 inhibition can effectively block EMT (Table 7).
Furthermore, the DNA methylation landscape is consistently altered during EMT. CpG island hypermethylation is seen in many promoters of genes essential in epithelial maintenance, associated with repression. In contrast, activation of EMT markers and stemness genes correlated with $\mathrm{CpG}$ island hypomethylation [Davalos et al., 2012; Pistore et al., 2017]. Treating cell lines with the hypomethylating agent 5 -azacytidine resulted in upregulation of the miRNA-200 family members, which can bind and degrade the mRNA levels of the ZEB EMT-TFs, and was associated with EMT reversal and increased therapeutic sensitivity [Chekhun et al., 2016]. However, treatments with DNA demethylating agents, like 5-azacytidine, on various in vitro and in vivo solid cancer models gave conflicting results of its effects on EMT and metastasis.

\section{Anti-EMT Targeting Strategies: Targeting Mesenchymal Cells and the ECM}

Instead of preventing EMT, a potential alternative anti-EMT therapeutic strategy is to target the mesenchymal cells themselves by inhibiting the functions of mesenchymal-specific proteins, for example, using bioactive compounds or monoclonal antibodies against vimentin, fibronectin, and $\mathrm{N}$-cadherin (Table 8), or by inducing mesenchymal CSCs to undergo MET [Pattabiraman et al., 2016]. These methods have been previously demonstrated to reduce invasion and secondary tumour formation [Tanaka et al., 2010; Thaiparambil et al., 2011; Pattabiraman et al., 2016]. But we have to keep in mind that also non-transformed mesenchymal stromal cells will be affected by this approach. Furthermore, forcing mesenchy- 
Table 8. Overview of clinical trials testing mesenchymal-specific protein inhibitors as anti-cancer therapy

\begin{tabular}{|c|c|c|c|c|}
\hline Drug & Target & $\begin{array}{l}\text { Development } \\
\text { stage }\end{array}$ & Cancer type & Trial identifier \\
\hline Monoclonal antibody & Fibronectin & Phase II & Breast, colorectal, lung and non-small cell lung cancer & NCT01125085 (CS) \\
\hline ADH-1 & $\mathrm{N}$-cadherin & Phase II & Adrenocortical carcinoma and non-small cell lung cancer & NCT00264433 (CS) \\
\hline Pritumumab & Vimentin & Phase II & Brain cancer & GDCT0217875 (CS) \\
\hline
\end{tabular}

Clinical trials were searched on the Pharma Intelligence Centre GlobalData database by performing a clinical trial search with fibronectin, $\mathrm{N}$-cadherin, and vimentin as target in the oncology therapy area and application of the drug as monotherapy. Only the most advanced clinical trials are shown. CS, completed successfully.

Table 9. Overview of clinical trials testing MMP2/9 inhibitors as anti-cancer therapy

\begin{tabular}{lllll}
\hline Drug & Target & $\begin{array}{l}\text { Development } \\
\text { stage }\end{array}$ & Cancer type & Trial identifier \\
\hline AE-941 & MMP2/9/12 & Phase III & Renal cell cancer & NCT00005995 (CS) \\
Andecaliximab & MMP9 & Phase I & Glioblastoma multiforme & NCT03631836 (P) \\
Glucosamine & MMP9 & Phase II & Breast cancer & GDCT0129146 (T) \\
Halofuginone hydrobromide & MMP2 & Phase I & Solid cancers & NCT00027677 (CU) \\
ORA-101 & MMP2/9 & Phase II & Non-small cell lung cancer & GDCT0179269 (CN) \\
$\begin{array}{l}\text { Prinomastat } \\
\text { Rebimastat }\end{array}$ & MMP2/9 & Phase III & Non-small cell lung cancer & NCT00004199 (CU) \\
Tanomastat & MMP1/2/7/9/14 & Phase II & Prostate cancer & NCT00040755 (CS) \\
Tigapotide triflutate & MMP2/3/9/13 & Phase III & (Non)-small cell lung cancer & GDCT0022508 (CN) \\
\hline
\end{tabular}

Clinical trials were searched on the Pharma Intelligence Centre GlobalData database by performing a clinical trial search with MMP2 and MMP9 as target in the oncology therapy area and application of the drug as monotherapy. Only the most advanced clinical trials are shown. CN, completed (no results available); CS, completed successfully; CU, completed unsuccessfully; MMP, matrix metalloproteinase; $\mathrm{P}$, planned; $\mathrm{T}$, terminated.

mal cells towards a more epithelial phenotype may promote colonisation and the formation of secondary metastases from cells that have already disseminated from the primary tumour.

Another option is by focussing on the interactions of cancer cells with the ECM. Multiple MMPs are upregulated upon induction of EMT [Miyoshi et al., 2004, 2005; Sun et al., 2008]. MMPs are calcium-dependent zinc-containing endopeptidases capable of degrading various ECM proteins, ensuring a pericellular proteolysis of the basement membrane components, thereby facilitating migration and invasion. MMPs and their inhibitors are extensively examined as potential anti-cancer drugs (Table 9). But also inversely, the composition of the ECM affects the behaviour of cells. For years, the ECM was considered as a mere support structure, however, it actually is a very dynamic compartment that modulates and regulates multiple cellular functions. Cancer cell-ECM interactions are mostly medi- ated via integrin signalling, which converts the mechanical cues of the ECM into biochemical signals, a process called mechanotransduction. One of the cues sensed by the integrins is the ECM stiffness. The integrins assemble several protein complexes which act as adaptor complexes such as Src and the focal adhesion kinase (FAK), controlling their cytoskeletal reorganisation and cell adhesion, survival, migration, and invasion [Legate and Fassler, 2009]. As the ECM is such an important player in cancer metastasis, several inhibitors targeting mechanotransduction have been developed and tested, with the integrins and FAK being the most targeted effectors. Inhibition of the integrin function has shown to lead to reduced metastatic burden in animal models and causes a prolonged stable disease state in clinical trials (Table 10). Targeting integrins in vivo is not easy as some clinical trials showed no significant improvement, which can be due to the broad function of these cell-ECM adhesion molecules. Numerous studies have focussed on 
Table 10. Overview of clinical trials testing FAK inhibitors as anti-cancer therapy

\begin{tabular}{|c|c|c|c|c|}
\hline Drug & Target & $\begin{array}{l}\text { Development } \\
\text { stage }\end{array}$ & Cancer type & Trial identifier \\
\hline AMP-945 & FAK & Phase I & Pancreatic and squamous cell carcinoma & GDCT0322541 (P) \\
\hline BI-853520 & FAK & Phase I & Pancreatic and ovarian cancer & NCT01335269 (CS) \\
\hline CEP-37440 & FAK & Phase I & Solid cancers & NCT01922752 (CN) \\
\hline CT-707 & FAK & Phase II & Non-small cell lung cancer & GDCT0335630 (P) \\
\hline Defactinib hydrochloride & & & Pancreatic cancer & NCT04331041 (P) \\
\hline GSK-2256098 & FAK & Phase I & $\begin{array}{l}\text { Breast, colon, gastric, liver, lung, ovarian, } \\
\text { prostate, rectal, and thyroid cancer }\end{array}$ & NCT01138033 (CS) \\
\hline MG-1102 & FAK & Phase I & Solid cancers & NCT01809912 (T) \\
\hline Repotrectinib & FAK & Phase I/II & $\begin{array}{l}\text { Gallbladder, liver, non-small cell lung, renal cell, } \\
\text { and thyroid cancer }\end{array}$ & NCT03093116 (O) \\
\hline
\end{tabular}

Clinical trials were searched on the Pharma Intelligence Centre GlobalData database by performing a clinical trial search with FAK as target in the oncology therapy area and application of the drug as monotherapy. Only the most advanced clinical trials are shown. $\mathrm{CN}$, completed (no results available); CS, completed successfully; FAK, focal adhesion kinase; O, ongoing; P, planned; T, terminated.

targeting the downstream pathway of the integrins, with the main focus on targeting FAK, with several FAK inhibitors showing promising anti-tumour effects in Phase I clinical trials (Table 11).

The final stages of the metastatic cascade may also provide a potential window for targeted therapies as cancer cells may be vulnerable during seeding and metastatic outgrowth. However, until now little is known on the exact molecular mechanisms controlling cancer cell colonisation and homing to distant sites. Multiple chemokine receptors have been demonstrated to be expressed on invasive cancer cells in a distinct and non-random pattern, whereas their ligands are expressed in organs that represent important sites of metastasis. Not only chemokine receptor blockers are being tested, also novel approaches are being developed to use these chemokines in transplantable and removable traps, sponges or scaffold that captures circulating cancer cells [Azarin et al., 2015; Najberg et al., 2019; Huang et al., 2020].

\section{In vitro and in vivo (Semi)High-Throughput Screening Tools to Identify Novel anti-EMT Compounds}

Unfortunately, many/most of the anti-EMT compounds that are available nowadays are broad-spectrum inhibitors and, as expected, they often have widespread side-effects. Most of them were identified under in vitro settings, using one or few transformed cell lines, often focussing on only one EMT-TF or a single upstream paracrine signal. The high complexity of the EMT regulatory network, variability of the cellular context and/or cellular microenvironment, and tissue heterogeneity in vivo are some of the hurdles to overcome. Because of this, many broad-spectrum inhibitors have failed in preclinical in vivo evaluation. As such, we are in need of more specific anti-EMT drugs. Extending our knowledge on the EMTTF network would help us to propose novel, more focussed and safer anti-EMT therapeutic strategies that can be used in a safe and effective manner in an in vivo setting and can be translated to clinical application. To identify these novel anti-EMT compounds, we need effective in vitro/in vivo semi-high throughput screening tools (Table 12).

The process of identifying compounds as potential EMT therapeutics requires the use of systems that are informative, unbiased, cost-effective, and fast - preferably automated. In vitro studies are often the first point of call in screening compounds for therapeutic potential. Classically, such studies have been performed in 2D systems using a combination of immunostaining, basic cell morphology, and migration assays to identify key cell markers of epithelial/mesenchymal character, morphology, and migratory/invasive capabilities. In more recent years, shRNA and CRISPR/Cas libraries have also been used to 
Table 11. Overview of clinical trials testing integrin inhibitors as anti-cancer therapy

\begin{tabular}{|c|c|c|c|c|}
\hline Abituzumab & ITGAV & Phase II & Prostate cancer & NCT01360840 (CS) \\
\hline ATN-161 & ITGA5/B1 & Phase II & Renal cell cancer & NCT00131651 (T) \\
\hline \multirow{2}{*}{ Cilengitide } & ITGAV & Phase II & Glioblastoma multiforme & NCT01782976 (T) \\
\hline & ITGB3/B5 & & Metastatic melanoma & NCT00082875 (T) \\
\hline \multirow[t]{2}{*}{ GLPG-0187 } & ITGA5/AV & Phase I & Solid cancer & NCT01580644 (CN) \\
\hline & ITGB1/B3/B5/B6 & & & NCT01313598 (CS) \\
\hline Intetumumab & ITGAV & Phase II & Prostate cancer & NCT00537381 (CU) \\
\hline & ITGB1/B3/B5/B6 & & & \\
\hline MK-0429 & ITGAV/B3 & Phase I & Prostate cancer & NCT00302471 (CS) \\
\hline PF-04605412 & ITGA5/B1 & Phase I & Solid cancers & NCT00915278 (T) \\
\hline
\end{tabular}

Clinical trials were searched on the Pharma Intelligence Centre GlobalData database by performing a clinical trial search with ITGA2B, ITGA5, ITGAV or ITGB1 as target in the oncology therapy area and application of the drug as monotherapy. Only the most advanced clinical trials are shown. CN, completed (no results available); CS, completed successfully; CU, completed unsuccessfully; ITGA, integrin alpha; ITGB, integrin beta; $\mathrm{T}$, terminated.

identify genes necessary for EMT activation as well as identifying genes upregulated specifically in chemoresistant cells [Raoof et al., 2019]. Due to the high-throughput nature of $2 \mathrm{D}$ in vitro studies, thousands of compounds can be screened in relatively short periods of time.

An example of such a screening was presented in a study in 2016, by Polireddy et al. [2016], screening 4 chemical libraries for their ability to inhibit EMT in the human pancreatic cancer cell line PANC-1. This screening method utilised the endogenous mesenchymal nature of PANC-1 cells to recapitulate populations of pancreatic CSCs in vitro. They used immunostaining to label membrane-bound E-cadherin. The mean fluorescence intensity was used as a quantitative readout for the epithelial character of cells. Compounds that induced high E-cadherin expression then underwent a second round of screening and were assayed for their ability to inhibit the migratory and tumour spheroid-forming capacity of both PANC-1 cells and an additional human pancreatic cell line BxPC-3. They found a single compound that reduced both migration and tumour spheroid formation of these cell lines; properties of cells that have undergone EMT and of CSCs, respectively, suggesting that high-throughput screening for compounds inhibiting EMT could be used to pull out compounds that also target CSCs. While not easily scalable to large high-throughput screenings, phenotypic, migratory, and spheroid formation assays are arguably far more informative when assessing whether cells have undergone an EMT relative to using basic epithelial/mesenchymal "markers," such as activation of E-cadherin expression. However, such an approach can either be used as a secondary screening as in the studies by Carmody et al. [2012], Gupta et al. [2009], and Polireddy et al. [2016], or for testing a more restricted number of compounds/candidate molecules.

$2 \mathrm{D}$ screenings have also been used to successfully identify the active compounds from traditional medicines; such as the EMT-inhibiting compound from within the Chinese herbal medicine "Tong-Mai-Yang-Xin-Wan" (TMYX). TMYX is widely used in treating cardiovascular diseases and other inflammation-related diseases and has been reported to prevent TGF- $\beta 1$-induced EMT characteristics in human kidney proximal tubule epithelial cells (HK-2) [Liu et al., 2016]. To identify which component of TMYX may inhibit EMT, 22 fractions of TMYX separated by liquid chromatography were applied to HK-2 cells treated with TGF- $\beta 1$ to stimulate EMT. Cells were assayed in microwell plates using automated microscopy for the induction of $\alpha$-smooth muscle actin ( $\alpha$-SMA), a biomarker associated with the mesenchymal character. Compounds isolated from the 5 fractions that were found to reduce expression of $\alpha$-SMA, were then further assayed in a secondary screening for their ability to induce E-cadherin expression [Liu et al., 2016]. This study assessed 


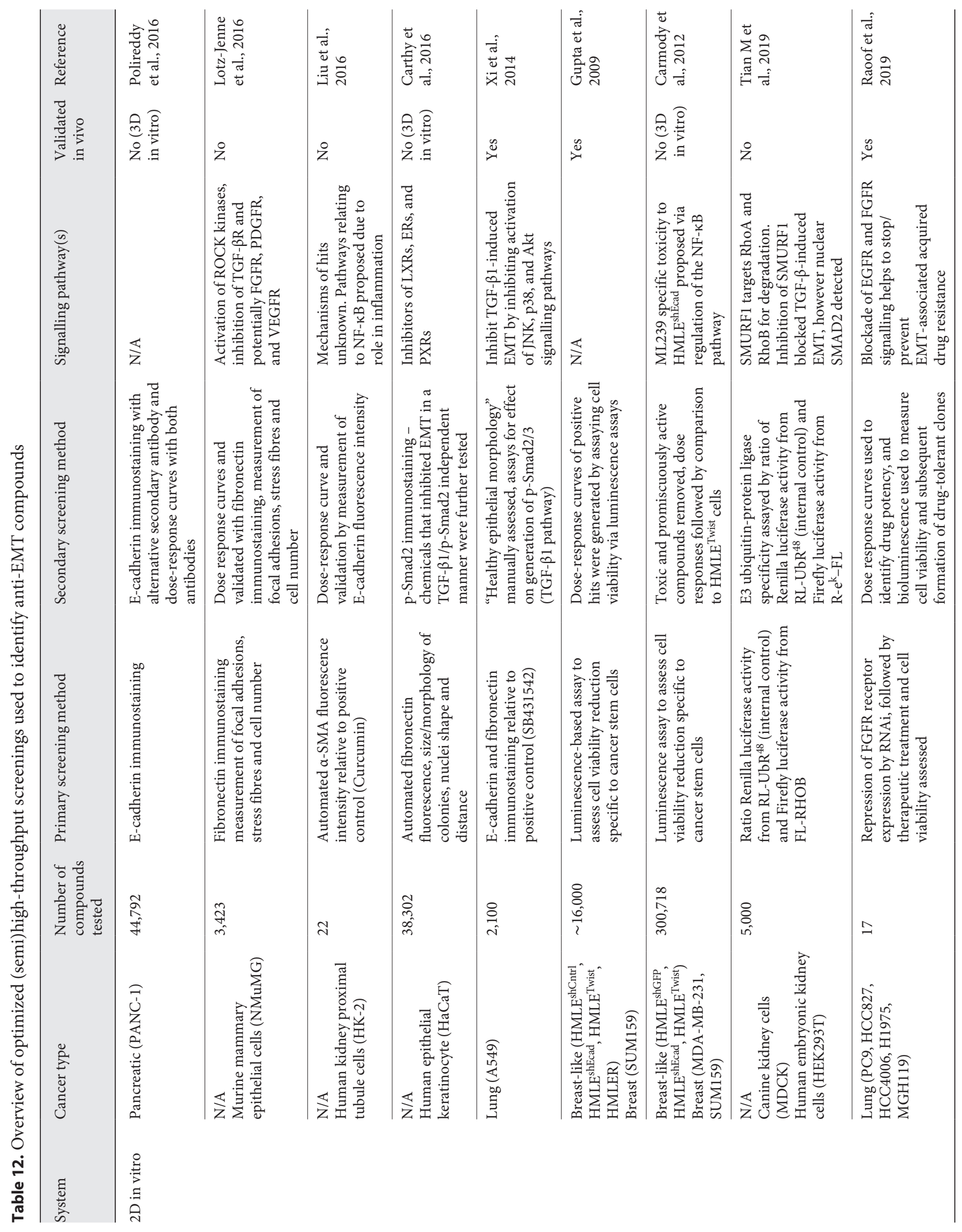




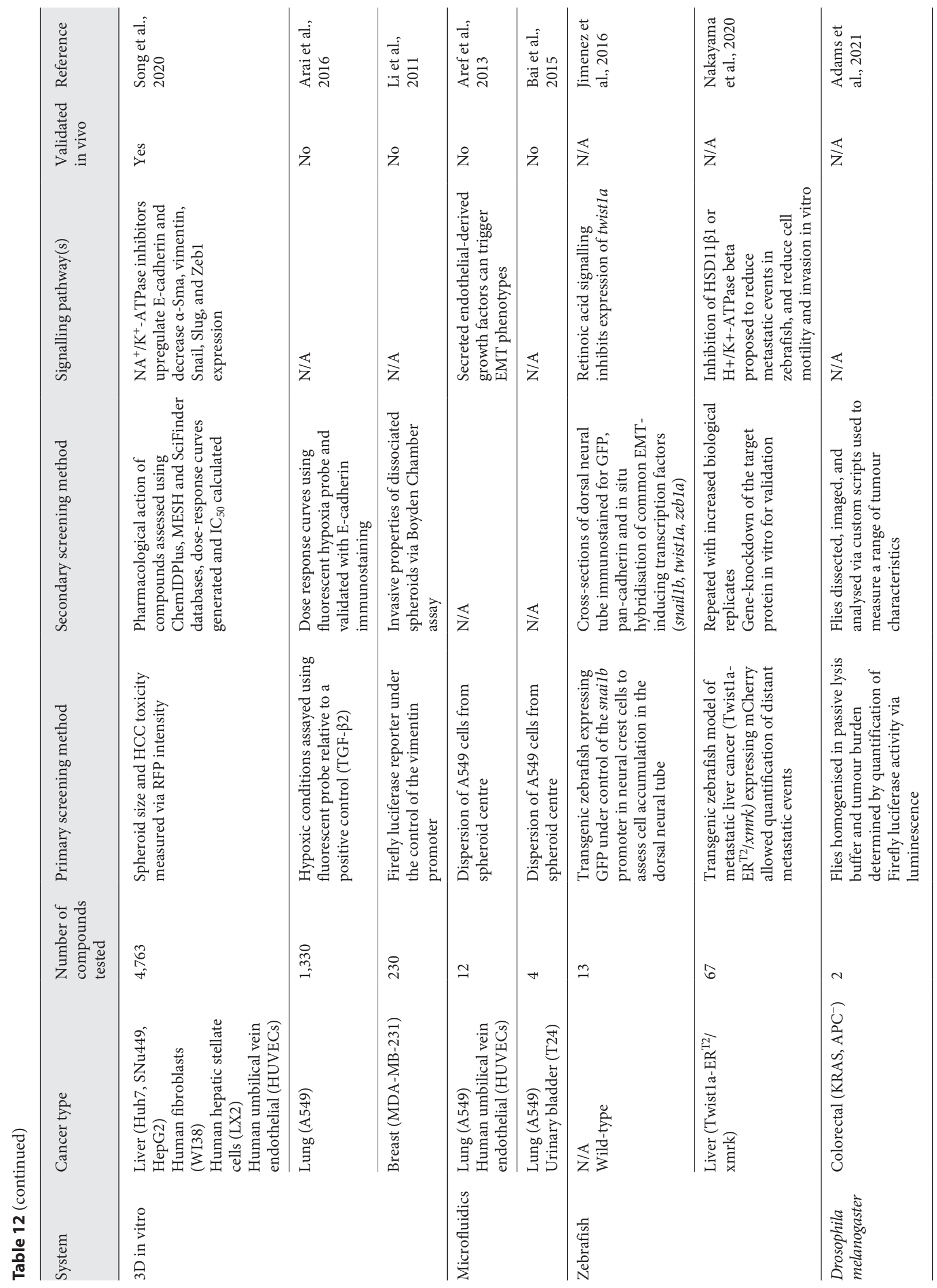


only 23 potential compounds that were narrowed down to 3 candidate therapeutic molecules. While this study by Liu et al. focussed on relatively few candidate compounds, the power of $2 \mathrm{D}$ in vitro assays allows the screening of up to thousands of chemicals, as demonstrated in similar screenings using fibronectin as a marker of mesenchymal character in keratinocytes [Carthy et al., 2016], or in murine mammary gland epithelial cells [Lotz-Jenne et al., 2016], or using both fibronectin and E-cadherin as markers for EMT in lung carcinoma cells [Xi et al., 2014].

The key advantages of 2D in vitro studies in therapeutic screening lie in an extremely fast turn-around, lowcost, the ease of manipulating cells in culture, and the array of readily available cell lines. However, the ease of use and fast turnaround times come at the cost of removing the rich biological environment found in vivo, a lack of targeted therapy delivery, and limited physical output (i.e., migration and invasion). This is particularly relevant for screenings targeting EMT, which has been shown to be highly influenced by cues that are absent in $2 \mathrm{D}$ cultures, such as the ECM [Poltavets et al., 2018], host stromal cells [Nowarski et al., 2017], and the mechanical environment [Nasrollahi and Pathak, 2016; Shellard and Mayor, 2019]. To overcome some of these pitfalls, 3D in vitro methods have been developed which enable the coculture of multiple cell types simultaneously [Jung et al., 2017; Lazzari et al., 2018], the culture of cells on an ECM [Rao Pattabhi et al., 2014; Singh and Tayalia, 2020], or scaffolds of varying stiffness [Matsuzaki et al., 2017; Rice et al., 2017], as well as the generation of tumour organoids [Drost and Clevers, 2018]. These techniques have enabled the generation of screening set-ups that more closely mimic an in vivo setting, while maintaining the highthroughput benefits of $2 \mathrm{D}$ in vitro screenings. Furthermore, the use of $3 \mathrm{D}$ cell culture allows the recapitulation of tumour properties that would be otherwise impossible to mimic in $2 \mathrm{D}$, such as the formation of a hypoxic environment at the centre of a tumour mass.

A novel approach to modelling tumours in 3D was taken by Arai et al. [2016], using specialised NanoCulture plates, containing nano-scale grid scaffolds on the base of the plate. This specialised scaffold reduces cell adhesion to the plate base and allows increased cell migration from scaffold to scaffold, promoting 3D spheroid formation. A combination of qRT-PCR, western blotting, live imaging, and immunostainings was used to characterise the behaviour of a variety of human carcinoma cell lines when plated on NanoCulture plates. The addition of TGF- $\beta$ to culture media induced an EMT programme, characterised by altered spheroid and cell morphology, increased cell budding and dissemination, and reduced frequencies of spheroid fusion events. Additionally, spheroid formation post-treatment was impacted. A key observation was that when the cells in this 3D model displayed epithelial traits, the spheroids were associated with increasingly hypoxic conditions which could be exploited as a high-throughput readout for the presence/absence of EMT. This was validated by a proof-of-concept screening of a library of 1,330 chemically active compounds, where they identified 2 components that were then shown to inhibit EMT in a number of follow-up assays.

Tumour spheroid formation has also been achieved via manipulation of physical properties of the surrounding environment. The alteration of synthetic ECM properties such as stiffness and cell-ECM adhesion has been shown to not only increase spheroid formation frequency, but also maintain cell proliferation and homeostasis and provide a potential model for testing chemoresistance. Such models, where an alternative in vitro environment is provided to promote $3 \mathrm{D}$ tumour formation, can be robustly used to analyse morphology and invasion/migration capabilities, rather than relying on simple epithelial/mesenchymal markers. Such techniques have greatly enhanced the screening capability of in vitro studies when testing compounds for anti-EMT potential, by reducing dependency on biological cell markers and allowing measurement of physiological traits known to be characteristic of an epithelial or mesenchymal nature [Li et al., 2011]. This is becoming important for assaying for EMT, as there are increasing studies demonstrating that EMT can lead to a number of intermediate epithelial/mesenchymal states that are not necessarily identifiably by classical markers such as a downregulation of E-cadherin or upregulation of $\alpha$-SMA or fibronectin [Pastushenko et al., 2018].

While recognition of the physical constraints provided by stromal tissue and ECM is important, stromal tissue also contains multiple cell types capable of secreting a variety of cytokines that may influence tumour progression. The "tumour-on-a-chip" technique attempts to model a number of the more complex interactions in vitro using a variety of cell types (epithelial, endothelial, stromal), tumour cells/spheroids, and a range of ECM constituents and physical constraints. Due to this wide range of possible variables, it has become possible to model a range of scenarios observed in vivo, as well as recapitulating individual stages within the metastatic cascade [SontheimerPhelps et al., 2019]. One such study utilised tumour-ona-chip technology to identify anti-EMT effects of chemical libraries when in the presence of tumour spheroids 
and cytokine secreting endothelial cells [Aref et al., 2013]. This study suspended tumour spheroids in a channel containing a synthetic ECM component in parallel to a channel containing human umbilical vein endothelial cells (HUVECs), both of whose growth is supported by the same culture medium. Cytokines secreted by the HUVEC monolayer can diffuse into the ECM-spheroid channel, and this has been demonstrated to induce EMT, resulting in dissociation of tumour spheroids [Jung et al., 2017]. Compounds can then be introduced into the spheroid channel and tested for their ability to block EMT through a variety of assays such as spheroid dissociation, relative dispersion of tumour cells, cell morphology, and immunostaining for epithelial/mesenchymal markers such as E-cadherin or vimentin [Aref et al., 2013]. The co-culture of multiple cell types and 3D culture techniques have led to a number of insights that potentially contribute to a number of issues found with therapeutics that show promise during in vitro tests but fail during in vivo testing. In a study by Bai et al. [2015], the co-culture of tumour spheroids with endothelial cell populations led to the identification of a reduction in efficacy of therapeutics due to the factors secreted by adjacent HUVECs.

The presence of stromal cells and ECM constituents allows for an increasingly accurate modelling of specific microenvironments in an in vitro setting. However, such in vitro studies still lack many biological elements present in vivo that are potentially key in promoting tumour progression, dissemination, and re-epithelialisation - for example, the immune system and the surrounding vasculature [De Palma et al., 2017; Janssen et al., 2017]. Additionally, such in vitro assays do not allow one to test if drug delivery is being targeted specifically to cancerous cells, without harming healthy tissues, which is an absolute requirement for any treatments to work in humans. Finally, while $3 \mathrm{D}$ in vitro techniques provide a basic view into modelling EMT, studying the potential effects blocking EMT may have on already circulating mesenchymal cells is not currently feasible. This aspect is potentially crucial when determining the viability of targeting EMT as a therapeutic target - if drugs targeting EMT promote MET in circulating mesenchymal cells they could actively promote the overt outgrowth of secondary metastases at distant sites - a potentially catastrophic side-effect. While this is extremely difficult to model in vitro, it is becoming increasingly possible to address in whole animal models.

While mouse models were viewed as the "gold-standard" of comparability when modelling human diseases such as cancer and have been instrumental in further val- idating many drugs first identified in in vitro screenings, they are not themselves amenable to high-, or even semihigh-throughput screenings [Frese and Tuveson, 2007; Rosenthal and Brown, 2007; Justice et al., 2011]. With drawbacks spanning experimental cost, the number of mice that would need to be sacrificed, and the time needed for such experiments limiting the efficacy of mice in high-throughput screening studies. Zebrafish are emerging as a powerful alternative model, whose use in highthroughput chemical screenings has been established for a range of cancers, including leukaemia, melanoma, and pancreatic cancer. They develop most of the organs found in mammals including those of the nervous, digestive, reproductive, immune, excretory, and cardiovascular systems [Shin and Fishman, 2002; Goldsmith and Jobin, 2012]. In contrast to mice, they develop rapidly, generate large numbers of progeny, and their maintenance cost is far lower.

The key role of EMT in developmental processes, such as the neural crest delamination and migration, can be exploited to screen for chemicals capable of blocking EMT. In an elegant screening, Jimenez et al. [2016] leveraged a transgenic zebrafish line they developed, in which the promoter from the EMT-TF Snailb is used to drive GFP. The reporter is turned on in neural crest cells prior to delamination from the neuroectoderm and maintained as the cells migrate. Zebrafish embryos were treated in a pilot chemical screening in 12-well plates, with 8-10 embryos in each well, and screened for EMT and migration defects using a fluorescence microscope, a process that could easily be up-scaled and automated. Interestingly, compounds found to have inhibitory effects on pathways previously shown to affect EMT in vitro, were ineffective in preventing neural crest cell EMT. However, they did find that addition of a compound, TSP-0903, that acts as a multi-kinase inhibitor dramatically blocked neural crest EMT and migration - which has now been initiated in Phase I clinical trials for acute myeloid leukaemia and advanced solid tumours. The requirement for a multi-kinase inhibitor suggests potential compensatory pathways of EMT induction in neural crest cells and highlights the importance of using an in vivo system when screening for compounds with anti-EMT potential. In order to establish a high-throughput chemical screening platform in a pathological model of EMT, Nakayama et al. [2020] generated a model of metastatic liver cancer in zebrafish. The group generated a transgenic zebrafish line using the readily available model for liver cancer (xmrk) in combination with Tamoxifen inducible overexpression of Twistla (Twist1a-ER ${ }^{\mathrm{T} 2}$ ). In order to verify this model, 
this group undertook a chemical screening to identify compounds with anti-metastatic properties - narrowing down a panel of 67 candidate compounds to 3 with potential anti-EMT capabilities [Tian M et al., 2019; Nakayama et al., 2020; Song et al., 2020].

While zebrafish have been used in small compound screenings for a range of processes associated with tumourigenesis and cancer progression, there is a distinct lack of research investigating compounds for their ability to block EMT. While zebrafish pose an excellent model for investigating the molecular mechanisms driving EMT during developmental processes, studying these same EMT-inducing mechanisms in pathological EMTs in zebrafish cancer models is far more challenging. The generation of cancer models in zebrafish often requires either transplant of tumourigenic material or heterozygous mutations in tumour suppressor genes alongside the addition of carcinogenic chemicals to reduce tumour formation time - it can take as long as 3-9 months for $>50 \%$ tumour formation in the absence of carcinogens [Feitsma and Cuppen, 2008; White et al., 2013]. Models of tumour formation in zebrafish which have been induced via carcinogens are often stochastic in organ of origin [Zhao et al., 2015], and if zebrafish present multiple tumours across organs, this presents challenges in determining whether tumours are primary tumours or secondary metastases.

Moreover, mammalian studies have shown the variety of EMT-inducing pathways that exist across different tumour types and tissues of origin [Banyard and Bielenberg, 2015; Stemmler et al., 2019]. This could generate confounding results if specific EMT-inducing pathways are blocked and others are not. Therefore, a pre- and post-therapeutic screening of individual zebrafish would be required to determine anti-EMT effects of compounds, which dramatically reduces the high-throughput nature of small compound screening. Due to the stochasticity or low-throughput methods of cancer generation in zebrafish, an invertebrate in vivo system with reproducible tumour phenotypes and metastatic potential may provide a more reliable and informative model for whole-animal high-throughput screening of anti-EMT compounds.

Drosophila melanogaster (Drosophila) are emerging as a powerful tool to investigate the basic cellular and molecular mechanisms underlying many disease processes including cancer [Gonzalez, 2013], with nearly $75 \%$ of human disease-causing genes believed to have a functional homologue in the fly [Pandey and Nichols, 2011]. The use of Drosophila in identifying key mutations in cancer was first explored as early as in the 1960s [Gateff and
Schneiderman, 1969, 1974]. Drosophila make an incredibly powerful platform for high-throughput screening, with compounds fed to the flies in their food, possessing genomic tractability and a lack of genetic redundancy. Additionally, they have a very fast generation time and low cost alongside an enormous wealth of genetic tools available. A wide range of Drosophila tumour models already exist, including epithelial tumours, organotypic models, and liquid tumours [Mirzoyan et al., 2019], which have been leveraged in a number of studies to identify therapeutic compounds reducing tumour burden [Edwards et al., 2011; Willoughby et al., 2013; Markstein et al., 2014; Levine and Cagan, 2016]. While Drosophila are an extremely strong and robust disease model, they are not without disadvantages. The trade-off for highthroughput capabilities comes at the cost of relatively fewer mammalian orthologues relative to murine models, along with reduced genetic redundancy, which is likely to play a part in complex diseases such as cancer. Furthermore, while many of the steps of the metastatic cascade are conserved in Drosophila, there are some important differences, including an open circulatory system and no adaptive immunity.

Studies of the basic biology underpinning metastasis in Drosophila had been limited for a long time by a lack of models where cells can be followed from the initiation of migration from the primary tumour, to seeding and growth of secondary tumours in adult organisms. This longstanding limitation was recently overcome, when the EMT-TF Snail was used to drive EMT in a benign colorectal cancer (CRC) model, which had previously been demonstrated to show remarkable similarities to human CRC [Martorell et al., 2014]. Expressing Snail in tumour cells leads to the formation of large secondary metastases at multiple distant sites in the body. Snail drives a partial EMT, which subsequently breaks through the basal lamina of the Drosophila midgut, undergoes a collective migration, and seeds polyclonal metastases [Campbell et al., 2019]. The entire process from primary tumour growth and cancer cell dissemination to growth of metastases takes place in vivo, and with cancer cells labelled with both GFP and luciferase reporters, one can easily visualise and rapidly assay for every step of the process. Notably, secondary metastases undergo MET and re-epithelialise over time. Thus, this presents a unique model in which anti-EMT compounds can be directly tested for their ability to promote MET and vice versa. Adams et al. [2021] have now generated pipelines for both a high-throughput method of small compound/therapeutic testing, and lowthroughput in-depth characterisation of tumour proper- 
ties and phenotypes, which can be applied to both the benign and metastatic CRC models. The combination of these benign and metastatic models of CRC with the high-throughput analysis pipeline provides the opportunity to assess the efficacy of drugs in reducing tumour burden and growth rate, as well as blocking processes integral to the metastatic cascade such as EMT (tumour cell dissemination), MET (colonisation of secondary tissues), migratory mechanisms of disseminated cells, and the generation of chemoresistance.

\section{Conclusions}

Our increasing understanding of the role of EMT in cancer has demonstrated that it is not only important in the metastatic spread of cancer cells, but also affects therapy resistance and the acquisition of CSC properties. As EMT and EMT-TFs are involved in many of these pivotal aspects of cancer progression, the interest to interfere with EMT as a therapeutic strategy is rapidly rising. Most of the currently available anti-EMT drugs are focussed on blocking upstream inducers of EMT. As the majority of these were originally identified in developmental biological studies, the anti-EMT drugs that are nowadays available, are broad-spectrum inhibitors, being non-specific to the role of EMT in cancer, and often causing adverse side-effects. This leads to the result that most of the drugs listed in Tables 1-11 are discovered in screenings performed by pharmaceutical companies based on the inhibition of proliferation. It is obvious that there is a need of more specific drugs to only intervene with EMT specifically in the context of cancer, creating the urge to invest in and design drug screens selectively targeting EMT rather than other malignant characteristics such as proliferation, as demonstrated by Chua et al. [2012].

Instead of targeting the upstream inducers, targeting the pivotal players of EMT, EMT-TFs and their co-factors, could result in more specific anti-EMT drugs. However, their redundant functions and the many feedback mechanisms between these EMT-TFs remain unclear. Further research is essential to obtain more insights in the regulation of cancer-associated EMT and identify the true dependency factors for EMT. Genetic deletion of Zeb1 was demonstrated to be sufficient to reduce metastatic burden and resistance to chemotherapy of a pancreatic cancer mouse model [Krebs et al., 2017]. These data clearly demonstrate that EMT-TFs can be valuable therapeutic targets. However according to conventional approaches, transcription factors are considered as undruggable. Nev- ertheless, advances in the field, for example, the introduction of small-molecule protein-protein interaction inhibitors and proteolysis-targeted chimaeras, have changed the definition of "druggable" over recent years. These novel avenues pave the way for novel therapeutic strategies also targeting transcription factors.

The increasing availability of models in which to screen chemicals for anti-EMT properties in a highthroughput format has also advanced the field. However, the bulk of these models remain in vitro which, while helpful in narrowing chemical libraries of tens of thousands of compounds, lack vital components necessary to recapitulate key steps in the metastatic cascade. The generation of in vivo models of metastatic cancers has aided in combatting challenges observed in 2D models, such as the incorporation of stromal cell populations and physical constraints. Further to this, the generation of a variety of cancer models in vivo provides the means to study various mechanisms of tumour progression and metastatic spread. This is likely to become increasingly important, as a number of reports have shown different cancers undergoing EMT through a variety of mechanisms, utilising different combinations of EMT-TFs and characteristics along the spectrum of EMT. Overall, these in vivo experiments have demonstrated the highly important notion of context-specificity when applying anti-EMT drugs.

In the perspective of this review, the role of EMT in immune recognition and anti-tumor response shaping should also be considered, especially in the context of the recent improvements in cancer treatment using immunotherapy. Many experimental observations provide clues to how mesenchymal-like carcinoma cells can contribute to immune escape [Chen et al., 2014; Dongre et al., 2017]. EMT can also act as a driver of activation of the immunosuppressive network within the tumour microenvironment. This not only points at promising EMT targets to consider for improving immunotherapy but also to the development of EMT markers predictive for immunotherapy response.

Together, the in this review discussed emerging therapies targeting EMT canonical pathways (TGF- $\beta$ R, Wnt, EGFR, EMT-TF), epigenetic drugs and metabolic alterations should also be seen as potential important perspectives to sensitize current immunotherapy approaches for advanced heterogenous tumours.

Finally, there is a high need for research to identify the (sub)types of cancer and specific conditions in which cancer patients will benefit from an anti-EMT therapy and how to combine it with the classical chemotherapies. It is expected that a personalized medicine approach 
would be best. Although our interest and knowledge on the importance and the molecular drivers of EMT in cancer progression have drastically improved, there is still a high need of further studies on how we could apply antiEMT drugs in the treatment of cancer patients.

\section{Conflict of Interest Statement}

The authors have no conflicts of interest to declare.

\section{Funding Sources}

S.J. is a predoctoral fellow with the Research Foundation Flanders (FWO). This work was supported by Strategic Basic Research (SBO; S008518 N), Stand up to Cancer (Kom op tegen Kanker) and the Belgian Federation Against Cancer (BFAC). K.C. is supported by a Welcome Trust/Royal Society Sir Henry Dale Fellowship (204615/Z/16/Z).

\section{Author Contributions}

S.G., G.B., and K.C. drafted the overall concept of this work. S.J., J.A., and S.G. wrote the review with help from D.D.G., K.C., and G.B. All authors have read, revised and gave final approval of the submitted version.

\section{References}

Adams J, Casali A, Campbell K. Methods to generate and assay for distinct stages of cancer metastasis in adult Drosophila melanogaster. Methods Mol Biol. 2021;2179:161-170.

Aiello NM, Maddipati R, Norgard RJ, Balli D, Li J, Yuan S, et al. EMT Subtype Influences Epithelial Plasticity and Mode of Cell Migration. Dev Cell. 2018 Jun;45(6):681-95.e4.

Arai K, Eguchi T, Rahman MM, Sakamoto R, Masuda N, Nakatsura T, et al. A Novel HighThroughput 3D Screening System for EMT Inhibitors: A Pilot Screening Discovered the EMT Inhibitory Activity of CDK2 Inhibitor SU9516. PLoS One. 2016;11(9):e0162394.

Aref AR, Huang RY, Yu W, Chua KN, Sun W, Tu TY, et al. Screening therapeutic EMT blocking agents in a three-dimensional microenvironment. Integr Biol (Camb). 2013 Feb;5(2): $381-9$.

Ayob AZ, Ramasamy TS. Cancer stem cells as key drivers of tumour progression. J Biomed Sci. 2018 Mar;25(1):20.

Azarin SM, Yi J, Gower RM, Aguado BA, Sullivan $\mathrm{ME}$, Goodman AG, et al. In vivo capture and label-free detection of early metastatic cells. Nat Commun. 2015 Sep;6:8094.

Bachman KE, Park BH. Duel nature of TGF-beta signaling: tumor suppressor vs. tumor promoter. Curr Opin Oncol. 2005 Jan; 17(1):4954.

Bai J, Tu TY, Kim C, Thiery JP, Kamm RD. Identification of drugs as single agents or in combination to prevent carcinoma dissemination in a microfluidic 3D environment. Oncotarget. 2015 Nov;6(34):36603-14.

Banyard J, Bielenberg DR. The role of EMT and MET in cancer dissemination. Connect Tissue Res. 2015;56(5):403-13.

Bao B, Wang Z, Ali S, Kong D, Banerjee S, Ahmad A, et al. Over-expression of FoxM1 leads to epithelial-mesenchymal transition and cancer stem cell phenotype in pancreatic cancer cells. J Cell Biochem. 2011 Sep;112(9):2296306.
Basu S, Cheriyamundath S, Ben-Ze'ev A. Cell-cell adhesion: linking Wnt/beta-catenin signaling with partial EMT and stemness traits in tumorigenesis. F1000Research. 2018;7:F1000.

Bennett CF, Swayze EE. RNA targeting therapeutics: molecular mechanisms of antisense oligonucleotides as a therapeutic platform. Annu Rev Pharmacol Toxicol. 2010;50:25993.

Bierie B, Moses HL. Transforming growth factor beta (TGF-beta) and inflammation in cancer. Cytokine Growth Factor Rev. 2010 Feb;21(1): 49-59.

Bogachek MV, Chen Y, Kulak MV, Woodfield GW, Cyr AR, Park JM, et al. Sumoylation pathway is required to maintain the basal breast cancer subtype. Cancer Cell. 2014 Jun; 25(6):748-61.

Boulding T, McCuaig RD, Tan A, Hardy K, Wu F, Dunn J, et al. LSD1 activation promotes inducible EMT programs and modulates the tumour microenvironment in breast cancer. Sci Rep. 2018 Jan;8(1):73.

Boumahdi S, de Sauvage FJ. The great escape: tumour cell plasticity in resistance to targeted therapy. Nat Rev Drug Discov. 2020 Jan; 19(1):39-56.

Bourcy M, Suarez-Carmona M, Lambert J, Francart ME, Schroeder H, Delierneux C, et al. Tissue Factor Induced by Epithelial-Mesenchymal Transition Triggers a Procoagulant State That Drives Metastasis of Circulating Tumor Cells. Cancer Res. 2016 Jul;76(14): 4270-82.

Bray SJ. Notch signalling: a simple pathway becomes complex. Nat Rev Mol Cell Biol. 2006 Sep;7(9):678-89.

Campbell K, Rossi F, Adams J, Pitsidianaki I, Barriga FM, Garcia-Gerique L, et al. Collective cell migration and metastases induced by an epithelial-to-mesenchymal transition in Drosophila intestinal tumors. Nat Commun. 2019 May;10(1):2311.
Cano A, Pérez-Moreno MA, Rodrigo I, Locascio A, Blanco MJ, del Barrio MG, et al. The transcription factor snail controls epithelial-mesenchymal transitions by repressing E-cadherin expression. Nat Cell Biol. 2000 Feb;2(2): 76-83.

Cao Z, Livas T, Kyprianou N. Anoikis and EMT: Lethal "Liaisons" during Cancer Progression. Crit Rev Oncog. 2016;21(3-4):155-68.

Capaccione KM, Pine SR. The Notch signaling pathway as a mediator of tumor survival. Carcinogenesis. 2013 Jul;34(7):1420-30.

Carmeliet P, Jain RK. Angiogenesis in cancer and other diseases. Nature. 2000 Sep;407(6801): 249-57.

Carmody LC, Germain AR, VerPlank L, Nag PP, Muñoz B, Perez JR, et al. Phenotypic highthroughput screening elucidates target pathway in breast cancer stem cell-like cells. J Biomol Screen. 2012 Oct;17(9):1204-10.

Carthy JM, Stöter M, Bellomo C, Vanlandewijck M, Heldin A, Morén A, et al. Chemical regulators of epithelial plasticity reveal a nuclear receptor pathway controlling myofibroblast differentiation. Sci Rep. 2016 Jul; 6:29868.

Chaffer CL, San Juan BP, Lim E, Weinberg RA. EMT, cell plasticity and metastasis. Cancer Metastasis Rev. 2016 Dec;35(4):645-54.

Chekhun VF, Borikun TV, Lukianova NY. Effect of 5-azacytidine on miRNA expression in human breast cancer cells with different sensitivity to cytostatics. Exp Oncol. 2016 Mar; 38(1):26-30.

Chen L, Gibbons DL, Goswami S, Cortez MA, Ahn YH, Byers LA, et al. Metastasis is regulated via microRNA-200/ZEB1 axis control of tumour cell PD-L1 expression and intratumoral immunosuppression. Nat Commun. 2014 Oct;5:5241.

Cheung KJ, Ewald AJ. A collective route to metastasis: Seeding by tumor cell clusters. Science. 2016 Apr;352(6282):167-9. 
Chua KN, Sim WJ, Racine V, Lee SY, Goh BC, Thiery JP. A cell-based small molecule screening method for identifying inhibitors of epithelial-mesenchymal transition in carcinoma. PLoS One. 2012;7(3):e33183.

Comijn J, Berx G, Vermassen P, Verschueren K, van Grunsven L, Bruyneel E, et al. The twohanded $\mathrm{E}$ box binding zinc finger protein SIP1 downregulates E-cadherin and induces invasion. Mol Cell. 2001 Jun;7(6):1267-78.

Connolly EC, Freimuth J, Akhurst RJ. Complexities of TGF- $\beta$ targeted cancer therapy. Int J Biol Sci. 2012;8(7):964-78.

Costanza B, Umelo IA, Bellier J, Castronovo V, Turtoi A. Stromal Modulators of TGF- $\beta$ in Cancer. J Clin Med. 2017 Jan;6(1):7.

Cruciat CM, Niehrs C. Secreted and transmembrane wnt inhibitors and activators. Cold Spring Harb Perspect Biol. 2013 Mar;5(3): a015081.

Davalos V, Moutinho C, Villanueva A, Boque R, Silva P, Carneiro F, et al. Dynamic epigenetic regulation of the microRNA-200 family mediates epithelial and mesenchymal transitions in human tumorigenesis. Oncogene. 2012 Apr;31(16):2062-74.

Davis FM, Stewart TA, Thompson EW, Monteith GR. Targeting EMT in cancer: opportunities for pharmacological intervention. Trends Pharmacol Sci. 2014 Sep;35(9):479-88.

De A. Wnt/Ca2+ signaling pathway: a brief overview. Acta Biochim Biophys Sin (Shanghai). 2011 Oct;43(10):745-56.

De Palma M, Biziato D, Petrova TV. Microenvironmental regulation of tumour angiogenesis. Nat Rev Cancer. 2017 Aug;17(8):457-74.

Derynck R, Budi EH. Specificity, versatility, and control of TGF-beta family signaling. Sci Signal. 2019 Feb;12(570):eaav5183.

Dongre A, Rashidian M, Reinhardt F, Bagnato A, Keckesova Z, Ploegh HL, et al. Epithelial-toMesenchymal Transition Contributes to Immunosuppression in Breast Carcinomas. Cancer Res. 2017 Aug;77(15):3982-9.

Drost J, Clevers H. Organoids in cancer research. Nat Rev Cancer. 2018 Jul;18(7):407-18.

Du B, Shim JS. Targeting Epithelial-Mesenchymal Transition (EMT) to Overcome Drug Resistance in Cancer. Molecules. 2016 Jul;21(7):965.

Edwards A, Gladstone M, Yoon P, Raben D, Frederick B, Su TT. Combinatorial effect of maytansinol and radiation in Drosophila and human cancer cells. Dis Model Mech. 2011 Jul; 4(4):496-503.

Fabregat I, Fernando J, Mainez J, Sancho P. TGFbeta signaling in cancer treatment. Curr Pharm Des. 2014;20(17):2934-47.

Feitsma H, Cuppen E. Zebrafish as a cancer model. Mol Cancer Res. 2008 May;6(5):685-94.

Fischer KR, Durrans A, Lee S, Sheng J, Li F, Wong ST, et al. Epithelial-to-mesenchymal transition is not required for lung metastasis but contributes to chemoresistance. Nature. 2015 Nov;527(7579):472-6.

Frese KK, Tuveson DA. Maximizing mouse cancer models. Nat Rev Cancer. 2007 Sep;7(9): 645-58.
Friberg S, Nyström A. Cancer Metastases: Early Dissemination and Late Recurrences. Cancer Growth Metastasis. 2015;8:43-9.

Friedl P, Alexander S. Cancer invasion and the microenvironment: plasticity and reciprocity. Cell. 2011 Nov;147(5):992-1009.

Fukagawa A, Ishii H, Miyazawa K, Saitoh M. $\delta$ EF1 associates with DNMT1 and maintains DNA methylation of the E-cadherin promoter in breast cancer cells. Cancer Med. 2015 Jan; $4(1): 125-35$.

Gateff E, Schneiderman HA. Neoplasms in mutant and cultured wild-tupe tissues of Drosophila. Natl Cancer Inst Monogr. 1969 Jul; 31:365-97.

Gateff E, Schneiderman HA. Developmental capacities of benign and malignant neoplasms of Drosophila. Wilhelm Roux Arch Entwickl Mech Org. 1974 Mar;176(1):23-65.

Genna A, Vanwynsberghe AM, Villard AV, Pottier C, Ancel J, Polette M, et al. EMT-Associated Heterogeneity in Circulating Tumor Cells: Sticky Friends on the Road to Metastasis. Cancers (Basel). 2020 Jun;12(6):1632.

Georgakopoulos-Soares I, Chartoumpekis DV, Kyriazopoulou V, Zaravinos A. EMT Factors and Metabolic Pathways in Cancer. Front Oncol. 2020;10:499.

Godin L, Balsat C, Van Eycke YR, Allard J, Royer C, Remmelink M, et al. A Novel Approach for Quantifying Cancer Cells Showing Hybrid Epithelial/Mesenchymal States in Large Series of Tissue Samples: Towards a New Prognostic Marker. Cancers (Basel). 2020 Apr; 12(4):906

Goldsmith JR, Jobin C. Think small: zebrafish as a model system of human pathology. J Biomed Biotechnol. 2012;2012:817341.

Gonzalez C. Drosophila melanogaster: a model and a tool to investigate malignancy and identify new therapeutics. Nat Rev Cancer. 2013 Mar;13(3):172-83.

Gupta PB, Onder TT, Jiang G, Tao K, Kuperwasser C, Weinberg RA, et al. Identification of selective inhibitors of cancer stem cells by high-throughput screening. Cell. 2009 Aug; 138(4):645-59.

Gupta S, Maitra A. EMT: Matter of Life or Death? Cell. 2016 Feb;164(5):840-2.

Haemmerle M, Stone RL, Menter DG, AfsharKharghan V, Sood AK. The Platelet Lifeline to Cancer: Challenges and Opportunities. Cancer Cell. 2018 Jun;33(6):965-83.

Hapach LA, Mosier JA, Wang W, Reinhart-King CA. Engineered models to parse apart the metastatic cascade. NPJ Precis Oncol. 2019;3: 20.

Hata A, Chen YG. TGF- $\beta$ Signaling from Receptors to Smads. Cold Spring Harb Perspect Biol. 2016 Sep;8(9):8.

Helbig G, Christopherson KW 2nd, BhatNakshatri P, Kumar S, Kishimoto H, Miller $\mathrm{KD}$, et al. NF-kappaB promotes breast cancer cell migration and metastasis by inducing the expression of the chemokine receptor CXCR4. J Biol Chem. 2003 Jun;278(24): 21631-8.
Heuberger J, Birchmeier W. Interplay of cadherin-mediated cell adhesion and canonical Wnt signaling. Cold Spring Harb Perspect Biol. 2010 Feb;2(2):a002915.

Hu W, Wang Z, Zhang S, Lu X, Wu J, Yu K, et al. IQGAP1 promotes pancreatic cancer progression and epithelial-mesenchymal transition (EMT) through Wnt/beta-catenin signaling. Sci Rep. 2019 May;9(1):7539.

Huang Y, Hakamivala A, Li S, Nair A, Saxena R, Hsieh JT, et al. Chemokine releasing particle implants for trapping circulating prostate cancer cells. Sci Rep. 2020 Mar;10(1):4433.

Husemann Y, Geigl JB, Schubert F, Musiani P, Meyer M, Burghart E, et al. Systemic spread is an early step in breast cancer. Cancer Cell. 2008 Jan;13(1):58-68.

Janssen LME, Ramsay EE, Logsdon CD, Overwijk WW. The immune system in cancer metastasis: friend or foe?. J Immunother Cancer. 2017 Oct:5(1):79.

Ji M, Lee EJ, Kim KB, Kim Y, Sung R, Lee SJ, et al. HDAC inhibitors induce epithelial-mesenchymal transition in colon carcinoma cells. Oncol Rep. 2015 May;33(5):2299-308.

Jiang GM, Wang HS, Zhang F, Zhang KS, Liu ZC, Fang R, et al. Histone deacetylase inhibitor induction of epithelial-mesenchymal transitions via up-regulation of Snail facilitates cancer progression. Biochim Biophys Acta. 2013 Mar;1833(3):663-71.

Jimenez L, Wang J, Morrison MA, Whatcott C, Soh KK, Warner S, et al. Phenotypic chemical screening using a zebrafish neural crest EMT reporter identifies retinoic acid as an inhibitor of epithelial morphogenesis. Dis Model Mech. 2016 Apr;9(4):389-400.

Jung HR, Kang HM, Ryu JW, Kim DS, Noh KH, Kim ES, et al. Cell Spheroids with Enhanced Aggressiveness to Mimic Human Liver Cancer In Vitro and In Vivo. Sci Rep. 2017 Sep; 7(1):10499.

Justice MJ, Siracusa LD, Stewart AF. Technical approaches for mouse models of human disease. Dis Model Mech. 2011 May;4(3):30510.

Kalluri R, Weinberg RA. The basics of epithelialmesenchymal transition. J Clin Invest. 2009 Jun;119(6):1420-8.

Kang H, Kim H, Lee S, Youn H, Youn B. Role of metabolic reprogramming in epithelial-mesenchymal transition (EMT). Int J Mol Sci. 2019 Apr;20(8):2042.

Kar R, Jha NK, Jha SK, Sharma A, Dholpuria S, Asthana N, et al. A "NOTCH" Deeper into the Epithelial-To-Mesenchymal Transition (EMT) Program in Breast Cancer. Genes. 2019 Nov; 10(12):961.

Kim HB, Myung SJ. Clinical implications of the Hippo-YAP pathway in multiple cancer contexts. BMB Rep. 2018 Mar;51(3):119-25.

Kim J, Kong J, Chang H, Kim H, Kim A. EGF induces epithelial-mesenchymal transition through phospho-Smad2/3-Snail signaling pathway in breast cancer cells. Oncotarget. 2016 Dec;7(51):85021-32. 
Kim MY, Oskarsson T, Acharyya S, Nguyen DX, Zhang XH, Norton L, et al. Tumor self-seeding by circulating cancer cells. Cell. 2009 Dec; 139(7):1315-26.

Komiya Y, Habas R. Wnt signal transduction pathways. Organogenesis. 2008 Apr;4(2):6875.

Kopan R. Notch signaling. Cold Spring Harb Perspect Biol. 2012 Oct;4(10):a011213.

Krebs AM, Mitschke J, Lasierra Losada M, Schmalhofer $\mathrm{O}$, Boerries $\mathrm{M}$, Busch $\mathrm{H}$, et al. The EMT-activator Zeb1 is a key factor for cell plasticity and promotes metastasis in pancreatic cancer. Nat Cell Biol. 2017 May;19(5): 518-29.

Kuhnert F, Kirshner JR, Thurston G. Dll4-Notch signaling as a therapeutic target in tumor angiogenesis. Vasc Cell. 2011 Sep;3(1):20.

Lamouille S, Xu J, Derynck R. Molecular mechanisms of epithelial-mesenchymal transition. Nat Rev Mol Cell Biol. 2014 Mar;15(3):17896.

Lazzari G, Nicolas V, Matsusaki M, Akashi M, Couvreur P, Mura S. Multicellular spheroid based on a triple co-culture: A novel 3D model to mimic pancreatic tumor complexity. Acta Biomater. 2018 Sep;78:296-307.

Legate KR, Fässler R. Mechanisms that regulate adaptor binding to beta-integrin cytoplasmic tails. J Cell Sci. 2009 Jan;122(Pt 2):187-98.

Lehmann W, Mossmann D, Kleemann J, Mock K, Meisinger C, Brummer T, et al. ZEB1 turns into a transcriptional activator by interacting with YAP1 in aggressive cancer types. Nat Commun. 2016 Feb;7:10498.

Levine BD, Cagan RL. Drosophila Lung Cancer Models Identify Trametinib plus Statin as Candidate Therapeutic. Cell Rep. 2016 Feb; 14(6):1477-87.

Li Q, Chen C, Kapadia A, Zhou Q, Harper MK, Schaack J, et al. 3D models of epithelial-mesenchymal transition in breast cancer metastasis: high-throughput screening assay development, validation, and pilot screen. J Biomol Screen. 2011 Feb;16(2):141-54.

Li Y, Seto E. HDACs and HDAC inhibitors in cancer development and therapy. Cold Spring Harb Perspect Med. 2016 Oct;6(10):a026831.

Lin G, Yu B, Liang Z, Li L, Qu S, Chen K, et al. Silencing of c-jun decreases cell migration, invasion, and EMT in radioresistant human nasopharyngeal carcinoma cell line CNE-2R. Onco Targets Ther. 2018;11:3805-15.

Ling HH, Kuo CC, Lin BX, Huang YH, Lin CW. Elevation of YAP promotes the epithelialmesenchymal transition and tumor aggressiveness in colorectal cancer. Exp Cell Res. 2017 Jan;350(1):218-25.

Liu N, Li L, Zhu X, Ling Z, Feng J, Hu Y, et al. A High Content Screening Assay to Identify Compounds with Anti-Epithelial-Mesenchymal Transition Effects from the Chinese Herbal Medicine Tong-Mai-Yang-Xin-Wan Molecules. 2016 Oct;21(10):1340.
Lotz-Jenne C, Lüthi U, Ackerknecht S, Lehembre F, Fink T, Stritt M, et al. A high-content EMT screen identifies multiple receptor tyrosine kinase inhibitors with activity on TGF $\beta$ receptor. Oncotarget. 2016 May; 7(18): 259836002.

MacDonald BT, He X. Frizzled and LRP5/6 receptors for Wnt $/ \beta$-catenin signaling. Cold Spring Harb Perspect Biol. 2012 Dec;4(12):a007880.

Maier HJ, Schmidt-Strassburger U, Huber MA, Wiedemann EM, Beug H, Wirth T. NF-kap$\mathrm{paB}$ promotes epithelial-mesenchymal transition, migration and invasion of pancreatic carcinoma cells. Cancer Lett. 2010 Sep;295(2): 214-28.

Maiques-Diaz A, Somervaille TC. LSD1: biologic roles and therapeutic targeting. Epigenomics. 2016 Aug;8(8):1103-16.

Majello B, Gorini F, Saccà CD, Amente S. Expanding the Role of the Histone Lysine-Specific Demethylase LSD1 in Cancer. Cancers (Basel). 2019 Mar;11(3):324.

Mani SA, Guo W, Liao MJ, Eaton EN, Ayyanan A, Zhou AY, et al. The epithelial-mesenchymal transition generates cells with properties of stem cells. Cell. 2008 May;133(4):704-15.

Markstein M, Dettorre S, Cho J, Neumüller RA, Craig-Müller S, Perrimon N. Systematic screen of chemotherapeutics in Drosophila stem cell tumors. Proc Natl Acad Sci USA. 2014 Mar;111(12):4530-5.

Martorell Ò, Merlos-Suárez A, Campbell K, Barriga FM, Christov CP, Miguel-Aliaga I, et al. Conserved mechanisms of tumorigenesis in the Drosophila adult midgut. PLoS One. 2014;9(2):e88413.

Massague J, Obenauf AC. Metastatic colonization by circulating tumour cells. Nature. 2016 Jan; 529(7586):298-306.

Matsuzaki S, Darcha C, Pouly JL, Canis M. Effects of matrix stiffness on epithelial to mesenchymal transition-like processes of endometrial epithelial cells: Implications for the pathogenesis of endometriosis. Sci Rep. 2017 Mar;7: 44616.

McDonald OG, Wu H, Timp W, Doi A, Feinberg AP. Genome-scale epigenetic reprogramming during epithelial-to-mesenchymal transition. Nat Struct Mol Biol. 2011 Jul;18(8): 867-74.

Meidhof S, Brabletz S, Lehmann W, Preca BT, Mock K, Ruh M, et al. ZEB1-associated drug resistance in cancer cells is reversed by the class I HDAC inhibitor mocetinostat. EMBO Mol Med. 2015 Jun;7(6):831-47.

Miele L. Notch signaling. Clin Cancer Res. 2006 Feb;12(4):1074-9.

Miller JR. The Wnts. Genome Biol. 2002;3(1):REVIEWS3001.

Mirzoyan Z, Sollazzo M, Allocca M, Valenza AM, Grifoni D, Bellosta P. Drosophila melanogaster: A Model Organism to Study Cancer. Front Genet. 2019;10:51.

Misra A, Pandey C, Sze SK, Thanabalu T. Hypoxia activated EGFR signaling induces epithelial to mesenchymal transition (EMT). PLoS One. 2012;7(11):e49766.
Miyoshi A, Kitajima Y, Sumi K, Sato K, Hagiwara A, Koga Y, et al. Snail and SIP1 increase cancer invasion by upregulating MMP family in hepatocellular carcinoma cells. Br J Cancer. 2004 Mar;90(6):1265-73.

Miyoshi A, Kitajima Y, Kido S, Shimonishi T, Matsuyama S, Kitahara K, et al. Snail accelerates cancer invasion by upregulating MMP expression and is associated with poor prognosis of hepatocellular carcinoma. Br J Cancer. 2005 Jan;92(2):252-8.

Mladinich M, Ruan D, Chan CH. Tackling Cancer Stem Cells via Inhibition of EMT Transcription Factors. Stem Cells Int. 2016;2016: 5285892.

Morel AP, Lièvre M, Thomas C, Hinkal G, Ansieau S, Puisieux A. Generation of breast cancer stem cells through epithelial-mesenchymal transition. PLoS One. 2008 Aug;3(8): e2888.

Mumm JS, Schroeter EH, Saxena MT, Griesemer A, Tian X, Pan DJ, et al. A ligand-induced extracellular cleavage regulates gamma-secretase-like proteolytic activation of Notch1. Mol Cell. 2000 Feb;5(2):197-206.

Nagrath S, Sequist LV, Maheswaran S, Bell DW, Irimia $\mathrm{D}$, Ulkus $\mathrm{L}$, et al. Isolation of rare circulating tumour cells in cancer patients by microchip technology. Nature. 2007 Dec; 450(7173):1235-9.

Najberg M, Haji Mansor M, Boury F, Alvarez-Lorenzo C, Garcion E. Reversing the Tumor Target: Establishment of a Tumor Trap. Front Pharmacol. 2019;10:887.

Nakayama J, Lu JW, Makinoshima H, Gong Z. A Novel Zebrafish Model of Metastasis Identifies the HSD11 $\beta 1$ Inhibitor Adrenosterone as a Suppressor of Epithelial-Mesenchymal Transition and Metastatic Dissemination. Mol Cancer Res. 2020 Mar;18(3):477-87.

Nasrollahi S, Pathak A. Topographic confinement of epithelial clusters induces epithelialto-mesenchymal transition in compliant matrices. Sci Rep. 2016 Jan;6:18831.

Niehrs C. The complex world of WNT receptor signalling. Nat Rev Mol Cell Biol. 2012 Dec; 13(12):767-79

Nieto MA, Huang RY, Jackson RA, Thiery JP. EMT: 2016. Cell. 2016 Jun;166(1661):21-45.

Normanno N, De Luca A, Bianco C, Strizzi L, Mancino M, Maiello MR, et al. Epidermal growth factor receptor (EGFR) signaling in cancer. Gene. 2006 Jan;366(1):2-16.

Nowarski R, Jackson R, Flavell RA. The Stromal Intervention: Regulation of Immunity and Inflammation at the Epithelial-Mesenchymal Barrier. Cell. 2017 Jan;168(3):362-75.

Olsauskas-Kuprys R, Zlobin A, Osipo C. Gamma secretase inhibitors of Notch signaling. Onco Targets Ther. 2013;6:943-55.

Palomero T, Lim WK, Odom DT, Sulis ML, Real $\mathrm{PJ}$, Margolin A, et al. NOTCH1 directly regulates c-MYC and activates a feed-forwardloop transcriptional network promoting leukemic cell growth. Proc Natl Acad Sci USA. 2006 Nov; 103(48):18261-6. 
Pandey UB, Nichols CD. Human disease models in Drosophila melanogaster and the role of the fly in therapeutic drug discovery. Pharmacol Rev. 2011 Jun;63(2):411-36.

Pasquier J, Abu-Kaoud N, Al Thani H, Rafii A. Epithelial to Mesenchymal Transition in a Clinical Perspective. J Oncol. 2015;2015: 792182.

Pastushenko I, Brisebarre A, Sifrim A, Fioramonti M, Revenco T, Boumahdi S, et al. Identification of the tumour transition states occurring during EMT. Nature. 2018 Apr;556(7702): 463-8.

Patel S, Alam A, Pant R, Chattopadhyay S. Wnt Signaling and Its Significance Within the Tumor Microenvironment: Novel Therapeutic Insights. Front Immunol. 2019;10:2872.

Pattabiraman DR, Bierie B, Kober KI, Thiru P, Krall JA, Zill C, et al. Activation of PKA leads to mesenchymal-to-epithelial transition and loss of tumor-initiating ability. Science. 2016 Mar;351(6277): aad3680.

Pistore C, Giannoni E, Colangelo T, Rizzo F, Magnani E, Muccillo L, et al. DNA methylation variations are required for epithelial-to-mesenchymal transition induced by cancer-associated fibroblasts in prostate cancer cells. Oncogene. 2017 Oct;36(40):5551-66.

Podsypanina K, Du YC, Jechlinger M, Beverly LJ, Hambardzumyan D, Varmus H. Seeding and propagation of untransformed mouse mammary cells in the lung. Science. 2008 Sep; 321(5897):1841-4.

Polireddy K, Dong R, McDonald PR, Wang T, Luke B, Chen P, et al. Targeting EpithelialMesenchymal Transition for Identification of Inhibitors for Pancreatic Cancer Cell Invasion and Tumor Spheres Formation. PLoS One. 2016;11(10):e0164811.

Poltavets V, Kochetkova M, Pitson SM, Samuel MS. The Role of the Extracellular Matrix and Its Molecular and Cellular Regulators in Cancer Cell Plasticity. Front Oncol. 2018;8:431.

Rajaram P, Chandra P, Ticku S, Pallavi BK, Rudresh KB, Mansabdar P. Epidermal growth factor receptor: Role in human cancer. Indian J Dent Res. 2017 Nov-Dec;28(6):687-94.

Rao Pattabhi S, Martinez JS, Keller TC 3rd. Decellularized ECM effects on human mesenchymal stem cell stemness and differentiation. Differentiation. 2014 Nov-Dec; 88(4冈5): 13143.

Raoof S, Mulford IJ, Frisco-Cabanos H, Nangia V, Timonina D, Labrot E, et al. Targeting FGFR overcomes EMT-mediated resistance in EGFR mutant non-small cell lung cancer. Oncogene. 2019 Sep;38(37):6399-413.

Reiss K, Saftig P. The "a disintegrin and metalloprotease" (ADAM) family of sheddases: physiological and cellular functions. Semin Cell Dev Biol. 2009 Apr;20(2):126-37.

Rice AJ, Cortes E, Lachowski D, Cheung BCH, Karim SA, Morton JP, et al. Matrix stiffness induces epithelial-mesenchymal transition and promotes chemoresistance in pancreatic cancer cells. Oncogenesis. 2017 Jul;6(7):e352.
Rosenthal N, Brown S. The mouse ascending: perspectives for human-disease models. Nat Cell Biol. 2007 Sep;9(9):993-9.

Salt MB, Bandyopadhyay S, McCormick F. Epithelial-to-mesenchymal transition rewires the molecular path to PI3K-dependent proliferation. Cancer Discov. 2014 Feb;4(2):18699.

Schlesinger M. Role of platelets and platelet receptors in cancer metastasis. J Hematol Oncol. 2018 Oct;11(1):125.

Sciacovelli M, Frezza C. Metabolic reprogramming and epithelial-to-mesenchymal transition in cancer. FEBS J. 2017 Oct;284(19): 3132-44.

Seshacharyulu P, Ponnusamy MP, Haridas D, Jain M, Ganti AK, Batra SK. Targeting the EGFR signaling pathway in cancer therapy. Expert Opin Ther Targets. 2012 Jan;16(1):1531.

Shaw HV, Koval A, Katanaev VL. Targeting the Wnt signalling pathway in cancer: prospects and perils. Swiss Med Wkly. 2019 Sep;149: w20129.

Shellard A, Mayor R. Integrating chemical and mechanical signals in neural crest cell migration. Curr Opin Genet Dev. 2019 Aug;57:1624.

Shen M, Xu Z, Xu W, Jiang K, Zhang F, Ding Q, et al. Inhibition of ATM reverses EMT and decreases metastatic potential of cisplatin-resistant lung cancer cells through JAK/STAT3/ PD-L1 pathway. J Exp Clin Cancer Res. 2019 Apr;38(1):149.

Shin JT, Fishman MC. From zebrafish to human: modular medical models. Annu Rev Genomics Hum Genet. 2002;3:311-40.

Sim WJ, Iyengar PV, Lama D, Lui SKL, Ng HC, Haviv-Shapira L, et al. c-Met activation leads to the establishment of a TGF $\beta$-receptor regulatory network in bladder cancer progression. Nat Commun. 2019 Sep;10(1):4349.

Singh A, Settleman J. EMT, cancer stem cells and drug resistance: an emerging axis of evil in the war on cancer. Oncogene. 2010 Aug;29(34): 4741-51.

Singh A, Tayalia P. Three-dimensional cryogel matrix for spheroid formation and anti-cancer drug screening. J Biomed Mater Res A. $2020 \mathrm{Feb} ; 108(2): 365-76$

Singh M, Yelle N, Venugopal C, Singh SK. EMT: Mechanisms and therapeutic implications. Pharmacol Ther. 2018 Feb;182:80-94.

Skrypek N, Goossens S, De Smedt E, Vandamme N, Berx G. Epithelial-to-Mesenchymal Transition: Epigenetic Reprogramming Driving Cellular Plasticity. Trends Genet. 2017 Dec; 33(12):943-59.

Song Y, Lee SY, Kim S, Choi I, Kim SH, Shum D, et al. Inhibitors of $\mathrm{Na}(+) / \mathrm{K}(+)$ ATPase exhibit antitumor effects on multicellular tumor spheroids of hepatocellular carcinoma. Sci Rep. 2020 Mar;10(1):5318.
Sonoshita M, Itatani Y, Kakizaki F, Sakimura K, Terashima T, Katsuyama Y, et al. Promotion of colorectal cancer invasion and metastasis through activation of NOTCH-DAB1-ABLRHOGEF protein TRIO. Cancer Discov. 2015 Feb;5(2):198-211.

Sontheimer-Phelps A, Hassell BA, Ingber DE. Modelling cancer in microfluidic human organs-on-chips. Nat Rev Cancer. 2019 Feb; 19(2):65-81.

Spaderna S, Schmalhofer O, Hlubek F, Berx G, Eger A, Merkel S, et al. A transient, EMTlinked loss of basement membranes indicates metastasis and poor survival in colorectal cancer. Gastroenterology. 2006 Sep;131(3): $830-40$.

Stemmler MP, Eccles RL, Brabletz S, Brabletz T. Non-redundant functions of EMT transcription factors. Nat Cell Biol. 2019 Jan;21(1): 102-12.

Stone RC, Pastar I, Ojeh N, Chen V, Liu S, Garzon KI, et al. Epithelial-mesenchymal transition in tissue repair and fibrosis. Cell Tissue Res. 2016 Sep;365(3):495-506.

Sun L, Diamond ME, Ottaviano AJ, Joseph MJ, Ananthanarayan V, Munshi HG. Transforming growth factor-beta 1 promotes matrix metalloproteinase-9-mediated oral cancer invasion through snail expression. Mol Cancer Res. 2008 Jan;6(1):10-20.

Syed V. TGF- $\beta$ Signaling in Cancer. J Cell Biochem. 2016 Jun;117(6):1279-87.

Tanaka H, Kono E, Tran CP, Miyazaki H, Yamashiro J, Shimomura T, et al. Monoclonal antibody targeting of $\mathrm{N}$-cadherin inhibits prostate cancer growth, metastasis and castration resistance. Nat Med. 2010 Dec;16(12): 1414-20.

Thaiparambil JT, Bender L, Ganesh T, Kline E, Patel P, Liu Y, et al. Withaferin A inhibits breast cancer invasion and metastasis at subcytotoxic doses by inducing vimentin disassembly and serine 56 phosphorylation. Int Cancer. 2011 Dec;129(11):2744-55.

Thiery JP, Acloque H, Huang RY, Nieto MA. Epithelial-mesenchymal transitions in development and disease. Cell. 2009 Nov;139(5):87190.

Tian M, Zeng T, Liu M, Han S, Lin H, Lin Q, et al. A cell-based high-throughput screening method based on a ubiquitin-reference technique for identifying modulators of $\mathrm{E} 3$ ligases. J Biol Chem. 2019 Feb;294(8):2880-91.

Tian T, Li X, Zhang J. mTOR Signaling in Cancer and mTOR Inhibitors in Solid Tumor Targeting Therapy. Int J Mol Sci. 2019 Feb;20(3):20.

Valastyan S, Weinberg RA. Tumor metastasis: molecular insights and evolving paradigms. Cell. 2011 Oct;147(2):275-92.

Verhoeven Y, Tilborghs S, Jacobs J, De Waele J, Quatannens D, Deben C, et al. The potential and controversy of targeting STAT family members in cancer. Semin Cancer Biol. 2020 Feb;60:41-56. 
Verstappen G, van Grunsven LA, Michiels C, Van de Putte T, Souopgui J, Van Damme J, et al. Atypical Mowat-Wilson patient confirms the importance of the novel association between ZFHX1B/SIP1 and NuRD corepressor complex. Hum Mol Genet. 2008 Apr;17(8):117583.

Wang Z, Li Y, Ahmad A, Azmi AS, Banerjee S, Kong $\mathrm{D}$, et al. Targeting Notch signaling pathway to overcome drug resistance for cancer therapy. Biochim Biophys Acta. 2010a Dec;1806(2):258-67.

Wang Z, Li Y, Kong D, Sarkar FH. The role of Notch signaling pathway in epithelial-mesenchymal transition (EMT) during development and tumor aggressiveness. Curr Drug Targets. 2010b Jun;11(6):745-51.

Weeraratna AT, Jiang Y, Hostetter G, Rosenblatt $\mathrm{K}$, Duray P, Bittner M, et al. Wnt5a signaling directly affects cell motility and invasion of metastatic melanoma. Cancer Cell. 2002 Apr; 1(3):279-88.

White R, Rose K, Zon L. Zebrafish cancer: the state of the art and the path forward. Nat Rev Cancer. 2013 Sep;13(9):624-36.

Williams ED, Gao D, Redfern A, Thompson EW. Controversies around epithelial-mesenchymal plasticity in cancer metastasis. Nat Rev Cancer. 2019 Dec;19(12):716-32.

Willoughby LF, Schlosser T, Manning SA, Parisot JP, Street IP, Richardson HE, et al. An in vivo large-scale chemical screening platform using Drosophila for anti-cancer drug discovery. Dis Model Mech. 2013 Mar;6(2):521-9.

Xi Y, Tan K, Brumwell AN, Chen SC, Kim YH, Kim TJ, et al. Inhibition of epithelial-to-mesenchymal transition and pulmonary fibrosis by methacycline. Am J Respir Cell Mol Biol. 2014 Jan;50(1):51-60.
Xia L, Tan S, Zhou Y, Lin J, Wang H, Oyang L, et al. Role of the NFkB-signaling pathway in cancer. Onco Targets Ther. 2018;11:2063-73.

Xia Y, Shen S, Verma IM. NF- $\mathrm{BB}$, an active player in human cancers. Cancer Immunol Res. 2014 Sep;2(9):823-30.

Xu Y, Lee D-K, Feng Z, Xu Y, Bu W, Li Y, et al. Breast tumor cell-specific knockout of Twist1 inhibits cancer cell plasticity, dissemination, and lung metastasis in mice. Proc Natl Acad Sci USA. 2017a Oct;114(43):11494-9.

Xu Y, Qin L, Sun T, Wu H, He T, Yang Z, et al. Twistl promotes breast cancer invasion and metastasis by silencing Foxa1 expression. Oncogene. 2017b Feb;36(8):115766.

Yang J, Mani SA, Donaher JL, Ramaswamy S, Itzykson RA, Come C, et al. Twist, a master regulator of morphogenesis, plays an essential role in tumor metastasis. Cell. 2004 Jun; 117(7):927-39.

Yang J, Nie J, Ma X, Wei Y, Peng Y, Wei X. Targeting PI3K in cancer: mechanisms and advances in clinical trials. Mol Cancer. $2019 \mathrm{Feb}$; 18(1):26.

Yang J, Antin P, Berx G, Blanpain C, Brabletz T, Bronner $\mathrm{M}$, et al. Guidelines and definitions for research on epithelial-mesenchymal transition. Nat Rev Mol Cell Biol. 2020 Jun;21(6): $341-52$.

Ye X, Weinberg RA. Epithelial-Mesenchymal Plasticity: A Central Regulator of Cancer Progression. Trends Cell Biol. 2015 Nov;25(11): 675-86.

Ye X, Brabletz T, Kang Y, Longmore GD, Nieto MA, Stanger BZ, et al. Upholding a role for EMT in breast cancer metastasis. Nature. 2017 Jul;547(7661):E1-3.

Yu M, Bardia A, Wittner BS, Stott SL, Smas ME, Ting DT, et al. Circulating breast tumor cells exhibit dynamic changes in epithelial and mesenchymal composition. Science. 2013 Feb;339(6119):580-4.
Yu Z, Pestell TG, Lisanti MP, Pestell RG. Cancer stem cells. Int J Biochem Cell Biol. 2012 Dec; 44(12):2144-51.

Yuan Y, Li D, Li H, Wang L, Tian G, Dong Y. YAP overexpression promotes the epithelial-mesenchymal transition and chemoresistance in pancreatic cancer cells. Mol Med Rep. 2016 Jan;13(1):237-42.

Zarzynska JM. Two faces of TGF-betal in breast cancer. Mediators Inflamm. 2014;2014: 141747.

Zhang J, Tian XJ, Xing J. Signal Transduction Pathways of EMT Induced by TGF- $\beta$, SHH, and WNT and Their Crosstalks. J Clin Med. 2016 Mar;5(4):41.

Zhang J, Zheng G, Zhou L, Li P, Yun M, Shi Q, et al. Notch signalling induces epithelialmesenchymal transition to promote metastasis in oral squamous cell carcinoma. Int J Mol Med. 2018 Oct;42(4):2276-84.

Zhang Y, Xia M, Jin K, Wang S, Wei H, Fan C, et al. Function of the c-Met receptor tyrosine kinase in carcinogenesis and associated therapeutic opportunities. Mol Cancer. 2018 Feb; 17(1):45.

Zhao S, Huang J, Ye J. A fresh look at zebrafish from the perspective of cancer research. J Exp Clin Cancer Res. 2015 Aug;34:80.

Zheng X, Carstens JL, Kim J, Scheible M, Kaye J, Sugimoto $\mathrm{H}$, et al. Epithelial-to-mesenchymal transition is dispensable for metastasis but induces chemoresistance in pancreatic cancer. Nature. 2015 Nov;527(7579):525-30.

Zhou Y, Liang C, Xue F, Chen W, Zhi X, Feng X, et al. Salinomycin decreases doxorubicin resistance in hepatocellular carcinoma cells by inhibiting the $\beta$-catenin/TCF complex association via FOXO3a activation. Oncotarget. 2015 Apr;6(12):10350-65. 